\title{
Unified formalism for electromagnetic and gravitational probes: Densities
}

\author{
Adam Freese $\odot^{*}$ and Gerald A. Miller $\oplus^{\dagger}$ \\ Department of Physics, University of Washington, Seattle, Washington, D.C. 98195, USA
}

(Received 2 September 2021; accepted 3 December 2021; published 4 January 2022)

\begin{abstract}
The use of light front coordinates allows a fully relativistic description of a hadron's spatial densities to be obtained. These densities must be two-dimensional and transverse to a chosen spatial direction. We explore their relationship to the three-dimensional, nonrelativistic densities, with a focus on densities associated with the energy-momentum tensor. The two-dimensional nonrelativistic densities can be obtained from the light front densities through a nonrelativistic limit, and can subsequently be transformed into three-dimensional nonrelativistic densities through an inverse Abel transform. However, this operation is not invertible, and moreover the application of the inverse Abel transform to the light front densities does not produce a physically meaningful result. We additionally find that the Abel transforms of so-called Breit-frame densities generally differ significantly from the light front densities. Numerical examples are provided to illustrate the various differences between the light front, Breit frame, and nonrelativistic treatment of densities.
\end{abstract}

DOI: 10.1103/PhysRevD.105.014003

\section{INTRODUCTION}

The energy-momentum tensor (EMT) and the associated gravitational form factors [1] have recently attracted significant interest in the hadron physics community. Major open questions such as the proton mass puzzle [2-8] and proton spin puzzle [9-13] are directly related to the EMT. Moreover, the EMT encodes information about the magnitude and distribution of forces within hadrons [14-17], a topic which has itself led to a flurry of theoretical studies [15,16], empirical extractions [18-20], and lattice calculations [21].

The theoretical studies are driven by the promise of making relevant experiments to determine the various matrix elements that allow the extraction of the relevant form factors. As depicted in Fig. 1, the relevant formalism is most generally expressed through generalized transverse momentum distributions, which are obtained from bilocal correlation function $H_{\Gamma}(k, P, \Delta)$ by integrating over $k^{-}$. For quarks, this correlator is given by [22]

$$
\begin{aligned}
H_{\Gamma}(k, P, \Delta)= & \frac{1}{(2 \pi)^{4}} \int \mathrm{d}^{4} z e^{i k \cdot z}\left\langle P+\frac{1}{2} \Delta\right| \bar{q}\left(-\frac{z}{2}\right) \\
& \times \Gamma \mathcal{W}\left(-\frac{z}{2}, \frac{z}{2}\right) q\left(\frac{z}{2}\right)\left|P-\frac{1}{2} \Delta\right\rangle,
\end{aligned}
$$

\footnotetext{
afreese@uw.edu

†miller@uw.edu
}

Published by the American Physical Society under the terms of the Creative Commons Attribution 4.0 International license. Further distribution of this work must maintain attribution to the author(s) and the published article's title, journal citation, and DOI. Funded by SCOAP. where $\Gamma$ stands in for a matrix in the Dirac algebra (e.g., $\gamma^{+}$) and $\mathcal{W}(y, x)$ is a Wilson line from $x$ to $y$. Integration over $\mathbf{k}_{\perp}$ gives the generalized parton distribution, Mellin moments of which encode local form factors of interestincluding those appearing in the EMT.

The form factors appearing in matrix elements of the EMT encode spatial densities via Fourier transforms. When performing these Fourier transforms, it is important to keep perspective about the actual, physical meaning of the densities that are obtained. It has been established $[17,23-26]$ that the only meaningful way to obtain fully relativistic densities is through two-dimensional Fourier transforms at fixed light front time. The three-dimensional Breit frame density was originally obtained by erroneously assuming that the hadron can be spatially localized [26,27], though it has been rehabilitated as a quasidensity through the Winger phase space formalism [16,28]. However, the phase space formalism of Refs. [16,28] sets $\mathbf{P}=0$ to obtain the Breit frame distributions, while integration over $\mathbf{P}$ is required to obtain physical expectation values from the Wigner distribution [29]. The Breit frame distributions are thus formally defined quantities.

The Abel transform has recently been proposed as a means of connecting the light front and Breit frame formalisms $[30,31]$. It is therefore necessary to explore the meaning of this connection. The Abel transform can be obtained by integrating one coordinate of a spherically symmetric density. However, there is no manifest spherical symmetry on the light front [32]. Additionally, Refs. [30,31] looked at the case of spin-half hadrons, but not spin-zero hadrons, where the proposed connection is shown below not to work.

The purpose here is to explore the actual meaning of 3D EMT densities and their relationship to the fully relativistic 


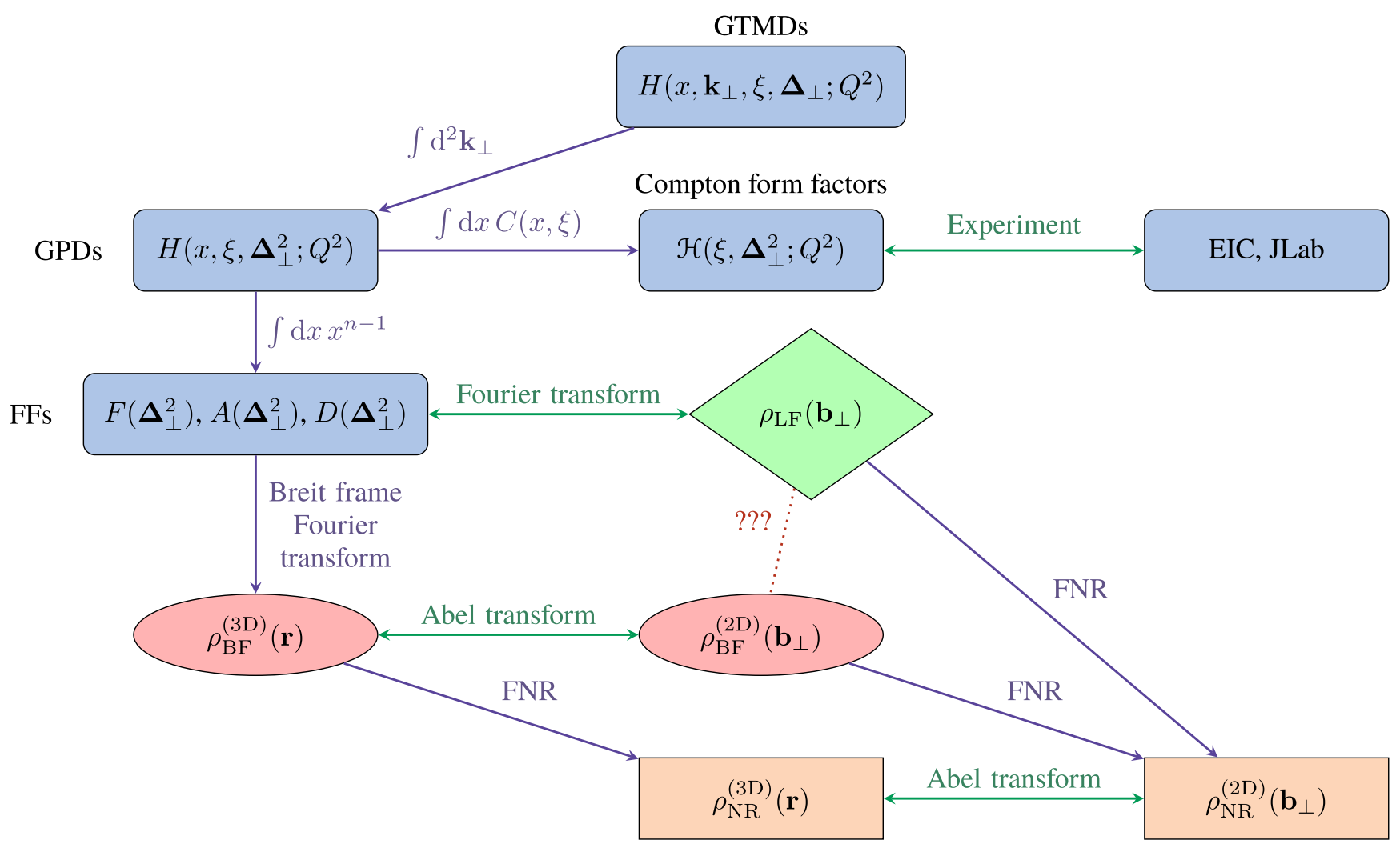

FIG. 1. Chart depicting the relationships between densities in different formalisms, as well as their relationships to generalized parton distributions. Here, $\rho$ stands in for any space-dependent density, including mass density, $P^{+}$density, or even pressure density. One-arrow (purple) lines signify one-way relationships, and two-arrow (green) lines signify invertible relationships. There is no systematic connection between light front and Breit frame densities.

2D light front densities. In particular, we show that physically meaningful 3D densities can be defined only in a nonrelativistic approximation, either by taking $c \rightarrow \infty$ or-in some cases-keeping up to order $v^{2} / c^{2}$ corrections. Additionally, we examine the physical meaning and applicability of the Abel transform.

This work is organized as follows. In Sec. II, we discuss the Abel transform and when it does and does not connect 2D and 3D densities. In Sec. III, we consider the formalism for relativistic and nonrelativistic densities for both spinzero and spin-half particles, deriving results for the relationships between them. Numerical examples, based on using a simple hadronic model $[25,33,34]$ are used to study the implications of using the Breit frame and the nonrelativistic limit in Sec. IV. We conclude and provide a summary in Sec. V.

\section{ABEL TRANSFORMS OF PHYSICAL DENSITIES}

Since the fully relativistic light front densities are twodimensional, they can be most directly compared to twodimensional rather than three-dimensional nonrelativistic densities. The 2D nonrelativistic densities are obtained by integrating out one coordinate of a given three-dimensional density $\rho_{\mathrm{NR}}^{(3 D)}(\mathbf{r})$ (which may be obtained as a three-dimensional Fourier transform of a form factor):

$$
\rho_{\mathrm{NR}}^{(2 D)}\left(\mathbf{b}_{\perp}\right)=\int_{-\infty}^{\infty} \mathrm{d} z \rho_{\mathrm{NR}}^{(3 D)}(\mathbf{r})
$$

where $\mathbf{b}_{\perp}=(x, y)$ are the transverse coordinates. If the 3D density is spherically symmetric, i.e., $\rho_{\mathrm{NR}}^{(3 D)}(\mathbf{r})$ is a function of only $r=|\mathbf{r}|$, a change of integration variable allows us to write

$\rho_{\mathrm{NR}}^{(2 D)}\left(b_{\perp}\right)=2 \int_{b}^{\infty} \mathrm{d} r \frac{r}{\sqrt{r^{2}-b_{\perp}^{2}}} \rho_{\mathrm{NR}}^{(3 D)}(r) \equiv \mathscr{A}\left[\rho_{\mathrm{NR}}^{(3 D)}(r)\right]\left(b_{\perp}\right)$,

which defines the Abel transform. ${ }^{1}$ For densities which depend on individual components of $\mathbf{r}$, one must use Eq. (2). However, each of the densities we consider can

\footnotetext{
${ }^{1}$ The Abel transform has several slightly different definitions in the literature. Equation (3) agrees with the definition in Ref. [35], which we use here because of its clear geometrical meaning. Reference [30] uses a different definition of the Abel transform.
} 
be written in terms of derivatives of an entirely scalar density to which Eq. (3) can be applied.

One pertinent property of the Abel transform is that it is invertible [35]:

$$
\begin{aligned}
\rho_{\mathrm{NR}}^{(3 D)}(r) & =-\frac{1}{\pi} \int_{r}^{\infty} \mathrm{d} b_{\perp} \frac{\rho_{\mathrm{NR}}^{(2 D)}\left(b_{\perp}\right)}{\mathrm{d} b_{\perp}} \frac{1}{\sqrt{b_{\perp}^{2}-r^{2}}} \\
& \equiv \mathscr{A}^{-1}\left[\rho_{\mathrm{NR}}^{(2 D)}\left(b_{\perp}\right)\right](r) .
\end{aligned}
$$

This allows a 3D nonrelativistic density to be reconstructed from a 2D nonrelativistic density, assuming that we know the former to be spherically symmetric ahead of time.

The importance of spherical symmetry cannot be stressed enough. If one begins with an azimuthally symmetric 2D density without a guarantee of spherical symmetry in three dimensions, applying Eq. (4) may not give the correct 3D density. Consider, for instance, the following 3D densities:

$$
\begin{aligned}
& f(r)=\frac{a^{3}}{\left(x^{2}+y^{2}+z^{2}+a^{2}\right)^{3}}, \\
& g(\mathbf{r})=\frac{s a^{3}}{\left(x^{2}+y^{2}+s^{2} z^{2}+a^{2}\right)^{3}},
\end{aligned}
$$

where $a$ is some length scale and $s$ is a positive unitless constant. These both integrate to the same azimuthally symmetric function:

$$
\begin{gathered}
\int_{-\infty}^{\infty} \mathrm{d} z f(r)=\int_{-\infty}^{\infty} \mathrm{d} z g(\mathbf{r})=\mathscr{A}[f(r)](b) \\
\equiv F(b)=\frac{3 a^{3} \pi}{8\left(a^{2}+b^{2}\right)^{5 / 2}} .
\end{gathered}
$$

Applying the inverse Abel transform to $F(b)$ will return $f(r)$, even if - in the context of a physical scenario- $g(\mathbf{r})$ is the true 3D density.

This point is especially pertinent since the light front Galilean subgroup of the Poincaré group does not have an $\mathrm{SO}(3)$ subgroup. Light front dynamics does not admit 3D spherical symmetry [32], so it is meaningless to try to construct an exact relativistic 3D density by applying Eq. (4) to a light front density, as done in Refs. [30,31] or earlier in Ref. [36]. In fact, there are model calculations suggesting that the proton is elongated in the $x^{-}$direction [37]. Moreover, we shall see below that densities of transversely polarized hadrons have $\phi$ dependence, demonstrating that spherical symmetry in $\left(\mathbf{x}_{\perp}, x^{-}\right)$does not hold. At best, the inverse Abel transform of a light front density can give the 3D density in a nonrelativistic approximation, as we shall show below.

One helpful property of Eq. (2) that will aid the exploration to follow is its effect on Fourier transforms. If a $3 \mathrm{D}$ density is defined by

$$
\rho_{\mathrm{NR}}^{(3 D)}(r)=\int \frac{\mathrm{d}^{3} \boldsymbol{\Delta}}{(2 \pi)^{3}} F\left(t=-\boldsymbol{\Delta}^{2}\right) e^{-i \boldsymbol{\Delta} \cdot \mathbf{r}},
$$

then because the $z$ integral of $e^{-i \Delta_{z} z}$ is $2 \pi \delta\left(\Delta_{z}\right)$, one has

$$
\rho_{\mathrm{NR}}^{(2 D)}\left(b_{\perp}\right)=\int \frac{\mathrm{d}^{2} \boldsymbol{\Delta}_{\perp}}{(2 \pi)^{2}} F\left(t=-\boldsymbol{\Delta}_{\perp}^{2}\right) e^{-i \boldsymbol{\Delta}_{\perp} \cdot \mathbf{b}_{\perp}} .
$$

\section{RELATIVISTIC AND NONRELATIVISTIC DENSITIES OF THE EMT}

We shall now consider relativistic and nonrelativistic densities of the energy-momentum tensor. These densities are related to form factors, which are defined via matrix elements of plane wave states. For spin-zero particles, the standard decomposition is [15]

$\left\langle p^{\prime}\left|T^{\mu \nu}(0)\right| p\right\rangle=2 P^{\mu} P^{\nu} A(t)+\frac{\Delta^{\mu} \Delta^{\nu}-\Delta^{2} g^{\mu \nu}}{2} D(t)$,

while for spin-half particles [15] it is

$$
\begin{aligned}
& \left\langle p^{\prime}, \lambda\left|T^{\mu \nu}(0)\right| p, \lambda\right\rangle \\
& =\bar{u}\left(p^{\prime}, \lambda\right)\left\{\frac{P^{\mu} P^{\nu}}{M} A(t)+\frac{\Delta^{\mu} \Delta^{\nu}-\Delta^{2} g^{\mu \nu}}{4 M} D(t)\right. \\
& \left.\quad+\frac{i P^{\{\mu} \sigma^{\nu\} \rho} \Delta_{\rho}}{2 M} J(t)\right\} u(p, \lambda),
\end{aligned}
$$

where $P=\frac{1}{2}\left(p+p^{\prime}\right), \Delta=p^{\prime}-p, t=\Delta^{2}$, and curly brackets \{\} signify symmetrization, e.g., $a^{\{\mu} b^{\nu\}}=a^{\mu} b^{\nu}+a^{\nu} b^{\mu}$.

\section{A. Relativistic light front densities}

As discussed in Refs. [23,24,32,38], the only way to meaningfully define intrinsic relativistic densities is at fixed light front time, since this allows separation between barycentric and relative coordinates. Although there is much emphasis in the literature on the concept of a probabilistic interpretation (pointing out that the transverse $j^{+}$and $T^{++}$densities correspond to probability densities), a probability interpretation is not necessary for the concept of a "density" to make physical sense. Here, we use the word "density" to refer to any physical quantity that is distributed over space, i.e., that can be defined via the expectation value

$$
\langle\Psi|\mathcal{O}(x)| \Psi\rangle
$$

for some local current $\mathcal{O}(x)$ and physical state $\Psi$. The true significance of light front coordinates, as first pointed out in Ref. [38] and later reiterated in Refs. [17,23,24], is that they allow a density defined at fixed light front time and integrated over $x^{-}$to be factorized into a center-of $-P^{+}$ piece convoluted with an intrinsic density, and for the latter 
to be isolated and well defined by taking the width of the barycentric wave function to zero.

For both spin-zero hadrons and longitudinally polarized spin-half hadrons, the light front momentum $\left(P^{+}\right)$density is found to be $[17,23,39]$

$$
\rho_{P^{+}}^{(\mathrm{LF})}\left(\mathbf{b}_{\perp}\right)=P^{+} \int \frac{\mathrm{d}^{2} \boldsymbol{\Delta}_{\perp}}{(2 \pi)^{2}} A(t) e^{-i \boldsymbol{\Delta}_{\perp} \cdot \mathbf{b}_{\perp}},
$$

and in these same cases, the comoving stress tensor is $[16,17]$

$S_{\mathrm{LF}}^{i j}\left(\mathbf{b}_{\perp}\right)=\frac{1}{4 P^{+}}\left(\delta^{i j} \nabla_{\perp}^{2}-\nabla_{\perp}^{i} \nabla_{\perp}^{j}\right) \int \frac{\mathrm{d}^{2} \boldsymbol{\Delta}_{\perp}}{(2 \pi)^{2}} D(t) e^{-i \boldsymbol{\Delta}_{\perp} \cdot \mathbf{b}_{\perp}}$.

The comoving stress tensor is a concept introduced in Ref. [17], and corresponds to what an observer comoving with the total motion of the hadron sees as the stress tensor. The full stress tensor depends on $\mathbf{P}_{\perp}$, and accordingly does not provide a well-defined density in the limit of localized wave packets. However, the Galilean symmetry allows the stress tensor to be additively decomposed into a piece that contains hadron flow and a piece that is invariant under Galilean boosts, and the latter piece-the comoving stress tensor-has no $\mathbf{P}_{\perp}$ dependence, and is well defined in the limit of localized wave packets. See Ref. [17] for a full explication of the formalism.

It should be emphasized that the stress tensor does not have a probability interpretation, since it mixes "good" and "bad" components of the fields, and accordingly contains contributions from particle pair production and annihilation [40]. This can be seen especially clearly in the formalism of generalized parton distributions, where the form factor $D(t)$ comes form the Efremov-Radyushkin-Brodsky-Lepage region [41], which connects Fock states with different numbers of particles [42]. The stress tensor is interpreted as a density, however, in the sense that it is a spatial distribution of a physical quantity, arising from the expectation value of a local current for a physical state.

The comoving stress tensor can be decomposed into a isotropic pressure $p^{\mathrm{LF}\left(b_{\perp}\right)}$ and pressure anisotropy (or shear stress function) $s^{\mathrm{LF}\left(b_{\perp}\right)}\left(b_{\perp}\right)$ :

$S_{\mathrm{LF}}^{i j}\left(\mathbf{b}_{\perp}\right)=\delta^{i j} p^{(\mathrm{LF})}\left(b_{\perp}\right)+\left(\frac{b_{\perp}^{i} b_{\perp}^{j}}{b_{\perp}^{2}}-\frac{1}{2} \delta^{i j}\right) s^{(\mathrm{LF})}\left(b_{\perp}\right)$,

and has eigenpressures in the tangential directions $[16,30,43]$ :

$p_{r}^{(\mathrm{LF})}\left(b_{\perp}\right)=\hat{b}_{i} \hat{b}_{j} S_{\mathrm{LF}}^{i j}\left(\mathbf{b}_{\perp}\right)=p^{(\mathrm{LF})}\left(b_{\perp}\right)+\frac{1}{2} s^{(\mathrm{LF})}\left(b_{\perp}\right)$,
$p_{t}^{(\mathrm{LF})}\left(b_{\perp}\right)=\hat{\phi}_{i} \hat{\phi}_{j} S_{\mathrm{LF}}^{i j}\left(\mathbf{b}_{\perp}\right)=p^{(\mathrm{LF})}\left(b_{\perp}\right)-\frac{1}{2} s^{(\mathrm{LF})}\left(b_{\perp}\right)$,

where $\hat{b}$ and $\hat{\phi}$ are unit vectors in the radial and tangential directions.

A useful quantity is the potential $\tilde{D}\left(b_{\perp}\right)$, defined by

$$
\tilde{D}\left(b_{\perp}\right)=\frac{1}{4 P^{+}} \int \frac{\mathrm{d}^{2} \boldsymbol{\Delta}_{\perp}}{(2 \pi)^{2}} D(t) e^{-i \boldsymbol{\Delta}_{\perp} \cdot \mathbf{b}_{\perp}} .
$$

The comoving stress tensor is related to this potential by

$$
S^{i j}\left(\mathbf{b}_{\perp}\right)=\left(\nabla^{i} \nabla^{j}-\delta^{i j} \nabla^{2}\right) \tilde{D}\left(b_{\perp}\right) .
$$

The radial and tangential pressures have simple expressions in terms of the potential:

$$
\begin{gathered}
p_{r}\left(b_{\perp}\right)=\frac{1}{b_{\perp}} \frac{\mathrm{d} \tilde{D}\left(b_{\perp}\right)}{\mathrm{d} b_{\perp}}, \\
p_{t}\left(b_{\perp}\right)=\frac{\mathrm{d}^{2} \tilde{D}\left(b_{\perp}\right)}{\mathrm{d} b_{\perp}^{2}} .
\end{gathered}
$$

\section{Transversely polarized hadrons}

It is possible to prepare spin-half hadrons in transversely polarized states, for which the light front densities will no longer have azimuthal symmetry. A transversely polarized hadron can be prepared as a superposition of light front helicity states [44]:

$$
\left|s_{T}=\mathbf{s}_{\perp}\right\rangle=\frac{|\lambda=+1\rangle+e^{i \phi_{s}}|\lambda=-1\rangle}{\sqrt{2}} .
$$

In terms of helicity states, matrix elements of transversely polarized states take the form (with momentum dependence suppressed to compactify the formula):

$$
\begin{aligned}
\left\langle\mathbf{s}_{\perp}|\hat{O}| \mathbf{s}_{\perp}\right\rangle= & \frac{1}{2}\left\{\langle+|\hat{O}|+\rangle+\langle-|\hat{O}|-\rangle+\langle+|\hat{O}|-\rangle e^{i \phi_{s}}\right. \\
& \left.+\langle-|\hat{O}|+\rangle e^{-i \phi_{s}}\right\},
\end{aligned}
$$

which is the average of helicity state densities, plus an additional $\phi$-dependent helicity-flip contribution.

The $P^{+}$density of transversely polarized states is

$$
\begin{aligned}
\rho_{P^{+}, T}^{(\mathrm{LF})}\left(\mathbf{b}_{\perp}, \mathbf{s}_{\perp}\right)= & \rho_{P^{+}}^{(\mathrm{LF})}\left(b_{\perp}\right)+P^{+} \frac{\sin (\phi)}{2 M c} \frac{\mathrm{d}}{\mathrm{d} b_{\perp}} \int \frac{\mathrm{d}^{2} \boldsymbol{\Delta}_{\perp}}{(2 \pi)^{2}} \\
& \times(A(t)-2 J(t)) e^{-i \boldsymbol{\Delta}_{\perp} \cdot \mathbf{b}_{\perp}},
\end{aligned}
$$

where $\rho_{P^{+}}^{(\mathrm{LF})}\left(b_{\perp}\right)$ is density for helicity states [equal to the spin-zero $P^{+}$density in Eq. (11)], and $\phi=\phi_{b}-\phi_{s}$ is the 
angle from the transverse polarization vector to the transverse coordinate. The result in Eq. (20) has previously been found in Refs. [23,39]. It is worth remarking that the angular dependence is a strictly relativistic effect because taking the $c \rightarrow \infty$ limit eliminates the $\phi$ dependence.

The comoving stress tensor also has angular dependence for transversely polarized states, and in addition has a new tensorial structure:

$$
\begin{aligned}
S_{T}^{i j}\left(\mathbf{b}_{\perp}, \mathbf{s}_{\perp}\right)= & \delta^{i j} p_{T}\left(b_{\perp}, \phi\right)+\left(\hat{b}^{i} \hat{b}^{j}-\frac{1}{2} \delta^{i j}\right) \\
& \times s_{T}\left(b_{\perp}, \phi\right)+\left(\hat{b}^{i} \hat{\phi}^{j}+\hat{b}^{j} \hat{\phi}^{i}\right) v_{T}\left(b_{\perp}\right), \\
p_{T}\left(b_{\perp}, \phi\right)= & p\left(b_{\perp}\right)+\frac{\sin (\phi)}{2 M c} p^{\prime}\left(b_{\perp}\right), \\
s_{T}\left(b_{\perp}, \phi\right)= & s\left(b_{\perp}\right)+\frac{\sin (\phi)}{2 M c} s^{\prime}\left(b_{\perp}\right), \\
v_{T}\left(b_{\perp}, \phi\right)= & \frac{\cos (\phi)}{2 M c} \frac{s\left(b_{\perp}\right)}{b_{\perp}}
\end{aligned}
$$

where $p\left(b_{\perp}\right)$ and $s\left(b_{\perp}\right)$ are the isotropic pressure and anisotropy functions in the helicity state case. The quantities $p_{T}, s_{T}, v_{T}$ cannot be obtained through an Abel transform.

The new tensor structure associated with $v_{T}\left(b_{\perp}, \phi\right)$ is peculiar and does not contribute to either the radial or the tangential pressure, since it contracts with both $\hat{b}^{i} \hat{b}^{j}$ and with $\hat{\phi}^{i} \hat{\phi}^{j}$ to zero. It is more instructive-as discussed in Ref. [15] — to find the eigenvalues and eigenvectors of the comoving stress tensor. Since the eigenvectors satisfy

$$
S_{T}^{i j}\left(\mathbf{b}_{\perp}, \mathbf{s}_{\perp}\right) \hat{e}_{ \pm}^{j}=p_{ \pm}\left(b_{\perp}, \phi\right) \hat{e}_{ \pm}^{i},
$$

where we use \pm to index the two eigenvectors (and their associated eigenpressures), it is possible to write the pressure in any direction as a superposition of the eigenpressures. If we write the eigenvectors in terms of an angle with respect to the transverse spin vector,

$$
\hat{e}_{ \pm}=\cos \left(\theta_{ \pm}\right) \mathbf{s}_{\perp}+\sin \left(\theta_{ \pm}\right) \tilde{\mathbf{s}}_{\perp},
$$

where $\tilde{\mathbf{s}}_{\perp}$ is a unit vector that is $90^{\circ}$ counterclockwise from $\mathbf{s}_{\perp}$, then the eigenvectors of $S_{T}^{i j}\left(\mathbf{b}_{\perp}, \mathbf{s}_{\perp}\right)$ are given by the angles

$$
\begin{aligned}
& \theta_{+}=\phi+\frac{1}{2} \tan ^{-1}\left(\frac{2 v_{T}\left(b_{\perp}, \phi\right)}{s_{T}\left(b_{\perp}, \phi\right)}\right) \\
& \theta_{-}=\phi+\frac{1}{2} \tan ^{-1}\left(\frac{2 v_{T}\left(b_{\perp}, \phi\right)}{s_{T}\left(b_{\perp}, \phi\right)}\right)+\frac{\pi}{2},
\end{aligned}
$$

while the associated eigenpressures are given by

$$
p_{ \pm}\left(b_{\perp}, \phi\right)=p_{T}\left(b_{\perp}, \phi\right) \pm \sqrt{\frac{1}{4}\left(s_{T}\left(b_{\perp}, \phi\right)\right)^{2}+\left(v_{T}\left(b_{\perp}, \phi\right)\right)^{2}}
$$

In the limit of large $b_{\perp}$, one has $v_{T}\left(b_{\perp}, \phi\right) \ll s_{T}\left(b_{\perp}, \phi\right)$, so the eigenangles become $\phi$ and $\phi+\frac{\pi}{2}$ very far from the center of the hadron. This deformation from the radial and tangential directions can be seen as a relativistic effect that vanishes in the $c \rightarrow \infty$ limit, along with the angular dependence of the eigenpressures.

The angular dependence in both the $P^{+}$density and the stress tensor demonstrates a lack of spherical symmetry in the light front formalism. This is of course not surprising, since rotations around the $x$ and $y$ axes are dynamical operators that do not commute with the light front Hamiltonian $P^{-}[32,45]$. This finding precludes use of the inverse Abel transform to construct a physically meaningful 3D relativistic density. Moreover, the inverse Abel transform cannot even be applied at a formal level, since the transform acts on an azimuthally symmetric function of a single variable.

\section{B. Breit frame densities}

If one tries to define 3D relativistic densities at fixed instant form time, the density becomes contaminated by center-of-mass motion of the hadron as a whole [27]. It is controversial whether localization of the center-of-mass motion in coordinate space is relativistically possible (see Refs. [46-48] for attempts, however), and localization in momentum space produces infinite radii for all densities [26] owing to the Heisenberg uncertainty principle.

Nonetheless, ostensibly relativistic 3D densities are ubiquitous throughout the hadron physics literature. The so-called Breit frame densities are defined by taking a Fourier transform of e.g., Eq. (9) with respect to the momentum transfer $\boldsymbol{\Delta}$ while setting the center-of-mass momentum to zero $(\mathbf{P}=0)$. This definition has been recently formalized in a Wigner phase space approach $[16,28]$, with $\mathbf{P}=0$ used to define the Breit frame distributions in particular. However, as explained for instance in Ref. [29], the physical expectation values for functions of space and momentum are obtained from a Wigner phase space distribution by integrating over both variables, rather than by setting $\mathbf{P}=0$. so the Breit frame distributions within this formalism therefore are definitions.

It is worth stressing that the Breit frame distributions have not been derived from the basic definition of a physical density, i.e., the expected value of a local current for a physical hadron state. As shown in Ref. [26], the original derivation in Ref. [49] was erroneous and neglected a term that would make all radii infinite. Nonetheless, the erroneous Breit frame densities with finite radii are ubiquitous enough that they should be addressed. 
The Breit frame mass density and stress tensor both have different expressions for spin-zero and spin-half particles. For the mass density $[14,15,50]$,

$$
\begin{aligned}
\rho_{\text {mass }}^{(j=0)}(\mathbf{r})= & M \int \frac{\mathrm{d}^{3} \boldsymbol{\Delta}}{(2 \pi)^{3}} \frac{1}{\sqrt{1-\frac{t}{4 M^{2}}}} \\
& \times\left\{A(t)-\frac{t}{4 M}[A(t)+D(t)]\right\} e^{-i \boldsymbol{\Delta} \cdot \mathbf{r}}, \quad(25 \mathrm{a}) \\
\rho_{\text {mass }}^{(j=1 / 2)}(\mathbf{r})= & M \int \frac{\mathrm{d}^{3} \boldsymbol{\Delta}}{(2 \pi)^{3}} \\
& \times\left\{A(t)-\frac{t}{4 M}[A(t)+D(t)-2 J(t)]\right\} e^{-i \boldsymbol{\Delta} \cdot \mathbf{r}},
\end{aligned}
$$

while for the stress tensor,

$$
\begin{aligned}
& T_{\mathrm{BF}, 0}^{i j}(\mathbf{r})=\frac{1}{4 M}\left(\delta^{i j} \boldsymbol{\nabla}^{2}-\nabla^{i} \boldsymbol{\nabla}^{j}\right) \int \frac{\mathrm{d}^{3} \boldsymbol{\Delta}}{(2 \pi)^{3}} \frac{1}{\sqrt{1-\frac{t}{4 M^{2}}}} D(t) e^{-i \Delta \cdot \mathbf{r}}, \\
& T_{\mathrm{BF}, \frac{1}{2}}^{i j}(\mathbf{r})=\frac{1}{4 M}\left(\delta^{i j} \nabla_{\perp}^{2}-\nabla_{\perp}^{i} \nabla_{\perp}^{j}\right) \int \frac{\mathrm{d}^{3} \boldsymbol{\Delta}}{(2 \pi)^{3}} D(t) e^{-i \Delta \cdot \mathbf{r}} .
\end{aligned}
$$

Using Eq. (8), it is possible to obtain simple formulas for the two-dimensional reductions of these Breit frame stress tensors. We find

$$
\begin{aligned}
T_{\mathrm{BF}, 0}^{i j}\left(\mathbf{b}_{\perp}\right)= & \frac{1}{4 M}\left(\delta^{i j} \nabla_{\perp}^{2}-\nabla_{\perp}^{i} \nabla_{\perp}^{j}\right) \int \frac{\mathrm{d}^{2} \boldsymbol{\Delta}_{\perp}}{(2 \pi)^{2}} \frac{1}{\sqrt{1-\frac{t}{4 M^{2}}}} \\
& \times D(t) e^{-i \boldsymbol{\Delta}_{\perp} \cdot \mathbf{b}_{\perp}} . \\
T_{\mathrm{BF}, \frac{1}{2}}^{i j}\left(\mathbf{b}_{\perp}\right)= & \frac{1}{4 M}\left(\delta^{i j} \nabla_{\perp}^{2}-\nabla_{\perp}^{i} \nabla_{\perp}^{j}\right) \int \frac{\mathrm{d}^{2} \boldsymbol{\Delta}_{\perp}}{(2 \pi)^{2}} D(t) e^{-i \boldsymbol{\Delta}_{\perp} \cdot \mathbf{b}_{\perp}},
\end{aligned}
$$

where the use of $\mathbf{b}_{\perp}$ instead of $\mathbf{r}$ is used to signify that these are $2 \mathrm{D}$ functions.

Comparing Eqs. (12) and (27b), one can see that $P^{+} S_{\mathrm{LF}}^{i j}\left(\mathbf{b}_{\perp}\right)=M T_{\mathrm{BF}, \frac{1}{2}}^{i j}\left(\mathbf{b}_{\perp}\right)$, i.e., that the $2 \mathrm{D}$ Breit frame and light front comoving stress tensors have identical forms (up to a constant) for spin-half particles specifically. This is essentially the central finding of Ref. [30]. The Abel transform connects 2D Breit frame densities to 3D Breit frame densities, and it just so happens that the Breit frame and light front comoving stress tensors have similar integrands specifically for spin-half particles. Because of this, Abel transforms formally work out to relate the 3D
Breit frame and 2D light front pressures for spin-half particles. It should be recalled however that the light front does not have 3D spherical symmetry and that the Breit frame densities are not physically meaningful densities. Thus this formal coincidence does not have any deep physical meaning, and does not lend credence to the Breit frame pressure.

By contrast, one can easily observe that $P^{+} S_{\mathrm{LF}}^{i j}\left(\mathbf{b}_{\perp}\right) \neq$ $M T_{\mathrm{BF}, 0}^{i j}\left(\mathbf{b}_{\perp}\right)$, so the findings of Ref. [30] do not apply to spin-zero particles. 3D Breit frame pressures in spin-zero hadrons are not related to 2D light front pressures by Abel transforms. In light of the caveats we have stressed so far, this is not surprising, but it does help stress that the findings of Ref. [30] originate from a coincidence rather than a deep connection between the light front and Breit frame.

\section{Nonrelativistic densities}

In a nonrelativistic (NR) quantum mechanical theory, just as in relativistic quantum field theory, the density associated with a local operator $\hat{\mathcal{O}}(x)$ and a physical state $|\Psi\rangle$ is given by

$$
\rho_{\mathrm{NR}}(\mathbf{r})=\langle\Psi|\hat{\mathcal{O}}(\mathbf{r})| \Psi\rangle_{\mathrm{NR}}
$$

The meaning of the term NR is that the system obeys Galilean invariance, in which the dependence on relative and center-of mass variables can be separated. The centerof-mass position of the physical state $|\Psi\rangle$ generally has a finite spatial extent. This state can be localized by allowing the total momentum to have infinite extent. This localization can be achieved, for example, by using a Gaussian representation [26] so that

$$
\Psi(\mathbf{p}, \mathbf{s})=(2 \pi)^{3 / 4}(2 \sigma)^{3 / 2} e^{-\sigma^{2} \mathbf{p}^{2}},
$$

in which $\mathbf{p}$ refers to the total momentum of the system, and then taking the $\sigma \rightarrow 0$ limit at the end of the calculation. The spatial dependence of $\rho_{\mathrm{NR}}(\mathbf{r})$ thus defined will encode only internal structure of the hadron. It is possible to show (using similar derivations to those in Refs. $[17,26]$ ) that the density can be written as

$$
\begin{aligned}
\rho_{\mathrm{NR}}(\mathbf{r})= & \lim _{\sigma \rightarrow 0}(2 \pi)^{3 / 2}(2 \sigma)^{3} \int \frac{\mathrm{d}^{3} \mathbf{P}}{(2 \pi)^{3}} \int \frac{\mathrm{d}^{3} \boldsymbol{\Delta}}{(2 \pi)^{3}} \\
& \times e^{-2 \sigma^{2} \mathbf{P}^{2}}\left\langle\mathbf{p}^{\prime}, \mathbf{s}|\hat{\mathcal{O}}(0)| \mathbf{p}, \mathbf{s}\right\rangle_{\mathrm{NR}} e^{-\frac{\sigma^{2}}{2} \boldsymbol{\Delta}^{2}} e^{-i \mathbf{\Delta} \cdot \mathbf{r}}
\end{aligned}
$$

in which $\mathbf{P}=\frac{1}{2}\left(\mathbf{p}+\mathbf{p}^{\prime}\right)$ and $\boldsymbol{\Delta}=\mathbf{p}^{\prime}-\mathbf{p}$. The limit $\sigma \rightarrow 0$ is to be taken after the $\mathbf{P}$ integral has been done. Integrals in which the matrix element contains factors $\mathbf{P}^{2}, \mathbf{P}^{4}$, etc. will diverge, which limits the densities that can be considered; for instance, we cannot calculate a kinetic energy density for a completely spatially localized system. 


\section{Nonrelativistic reduction}

The matrix element $\left\langle\mathbf{p}^{\prime}, \mathbf{s}|\hat{\mathcal{O}}(0)| \mathbf{p}, \mathbf{s}\right\rangle_{\mathrm{NR}}$ appearing in Eq. (30) is a nonrelativistic matrix element. ${ }^{2}$ In practice, one knows how to express the relativistic counterpart $\left\langle\mathbf{p}^{\prime}, \mathbf{s}|\hat{\mathcal{O}}(0)| \mathbf{p}, \mathbf{s}\right\rangle_{\text {rel }}$ in terms of local form factors. It should be possible to obtain the former from the latter by restoring factors of $c$ where appropriate and taking the $c \rightarrow \infty$ limit. Before doing so, we also must bear in mind that the momentum kets are normalized differently in the relativistic and nonrelativistic cases; the conventional (instant form) normalization for momentum kets is

$$
\begin{aligned}
\left\langle\mathbf{p}^{\prime}, \mathbf{s}^{\prime} \mid \mathbf{p}, \mathbf{s}\right\rangle_{\mathrm{NR}} & =(2 \pi)^{3} \delta^{(3)}\left(\mathbf{p}^{\prime}-\mathbf{p}\right) \delta_{s s^{\prime}}, \\
\left\langle\mathbf{p}^{\prime}, \mathbf{s}^{\prime} \mid \mathbf{p}, \mathbf{s}\right\rangle_{\mathrm{rel}} & =(2 \pi)^{3}\left(2 E_{\mathbf{p}}\right) \delta^{(3)}\left(\mathbf{p}^{\prime}-\mathbf{p}\right) \delta_{s s^{\prime}}
\end{aligned}
$$

Thus, the fully nonrelativistic (FNR) limit is given by

$$
\left\langle\mathbf{p}^{\prime}, \mathbf{s}|\hat{\mathcal{O}}(0)| \mathbf{p}, \mathbf{s}\right\rangle_{\mathrm{NR}}=\lim _{c \rightarrow \infty} \frac{1}{\sqrt{2 E_{\mathbf{p}} 2 E_{\mathbf{p}^{\prime}}}}\left\langle\mathbf{p}^{\prime}, \mathbf{s}|\hat{\mathcal{O}}(0)| \mathbf{p}, \mathbf{s}\right\rangle_{\text {rel }}
$$

Of course, one can take $E_{\mathbf{p}} / c^{2} \rightarrow M$ in the FNR limit, but in some cases it may be instructive to know what the leading relativistic corrections look like. These can be found by expanding the right-hand side of Eq. (32) as a power series in $|\mathbf{p}| /(M c)$, and dropping terms beyond a certain order instead of taking the $c \rightarrow \infty$ limit.

When taking the nonrelativistic limit, consistency demands that this limit be applied to the whole of the right-hand side of Eq. (32). For instance, in the case of the electric charge density of a spin-zero hadron, one has

$$
\begin{aligned}
\left\langle\mathbf{p}^{\prime}, \mathbf{s}\left|j^{0}(0)\right| \mathbf{p}, \mathbf{s}\right\rangle_{\mathrm{NR}} & =\lim _{c \rightarrow \infty}\left\{\frac{\left(E_{\mathbf{p}}+E_{\mathbf{p}^{\prime}}\right)}{\sqrt{2 E_{\mathbf{p}^{2}} 2 E_{\mathbf{p}^{\prime}}}} F(t)\right\} \\
& =\lim _{c \rightarrow \infty} F(t) \equiv F_{\mathrm{NR}}\left(t_{\mathrm{NR}}=-\boldsymbol{\Delta}^{2}\right) .
\end{aligned}
$$

In the nonrelativistic limit, the zeroth component of the four-momentum transfer $\Delta^{\mu}$ is $\mathcal{O}(1 / c)$ and is thus neglected, and the argument of the nonrelativistic form factor becomes $t_{\mathrm{NR}} \equiv-\Delta^{2}$. Since the dynamics that govern the structure of hadrons are manifestly relativistic, the form factor $F(t)$ will change in the nonrelativistic limit, as seen for instance in Ref. [25]. Consistent application of the nonrelativistic limit thus means that the form factors appearing in nonrelativistic 3D densities and fully relativistic light front densities should be different functions. We shall subscript the latter using NR.

\section{Nonrelativistic mass density}

In the nonrelativistic formalism, matrix elements of $T^{00}$ provide the mass density. For spin-zero and spin-half particles, respectively, we have

$$
\begin{aligned}
\left\langle\mathbf{p}^{\prime}, \mathbf{s}\left|T^{00}(0)\right| \mathbf{p}, \mathbf{s}\right\rangle_{\mathrm{NR}}^{(j=0)} \approx & M c^{2}\left\{\left[1+\frac{\mathbf{P}^{2}}{2 M^{2} c^{2}}+\frac{\boldsymbol{\Delta}^{2}}{8 M^{2} c^{2}}\right] A_{\mathrm{NR}}(t)+\frac{\boldsymbol{\Delta}^{2}}{4 M^{2} c^{2}} D_{\mathrm{NR}}(t)+\mathcal{O}\left(1 / c^{4}\right)\right\}, \\
\left\langle\mathbf{p}^{\prime}, \mathbf{s}\left|T^{00}(0)\right| \mathbf{p}, \mathbf{s}\right\rangle_{\mathrm{NR}}^{(j=1 / 2)} \approx & M c^{2}\left\{\left[1+\frac{\mathbf{P}^{2}}{2 M^{2} c^{2}}+\frac{\boldsymbol{\Delta}^{2}}{4 M^{2} c^{2}}-\frac{i(\boldsymbol{\Delta} \times \mathbf{P}) \cdot \mathbf{s}}{4 M^{2} c^{2}}\right] A_{\mathrm{NR}}(t)\right. \\
& \left.+\frac{\boldsymbol{\Delta}^{2}}{4 M^{2} c^{2}} D_{\mathrm{NR}}(t)+\left[-\frac{\boldsymbol{\Delta}^{2}}{2 M^{2} c^{2}}+\frac{i(\boldsymbol{\Delta} \times \mathbf{P}) \cdot \mathbf{s}}{M^{2} c^{2}}\right] J_{\mathrm{NR}}(t)+\mathcal{O}\left(1 / c^{4}\right)\right\},
\end{aligned}
$$

where the NR subscripts on the form factors indicate that they should be expanded in powers of $1 / c$ as well, and where here we use $|\mathbf{s}|=1$ for simplicity. Because of the $\mathbf{P}^{2}$ terms, these cannot be used to define densities for an arbitrarily localized hadron at order $1 / c^{2}$, though the $c \rightarrow \infty$ limit does not have this issue. However, if this matrix element is placed into Eq. (30) without taking the $\sigma \rightarrow 0$ limit, one obtains results in the form

$$
\rho_{\text {energy }}(\mathbf{r} ; \sigma)=\frac{\rho_{\text {mass }}(\mathbf{r} ; \sigma)}{M}\left\{M c^{2}+\frac{\left\langle\mathbf{P}^{2}\right\rangle_{\sigma}}{2 M}\right\}+\mathcal{O}\left(1 / c^{2}\right),
$$

where

$$
\begin{aligned}
\left\langle\mathbf{P}^{2}\right\rangle_{\sigma} & =\int \frac{\mathrm{d}^{3} \mathbf{P}}{(2 \pi)^{3}} \mathbf{P}^{2}|\psi(\mathbf{P}, \mathbf{s} ; \sigma)|^{2}=\frac{3}{\sigma^{2}}, \\
\rho_{\text {mass }}^{(j=0)}(\mathbf{r} ; \sigma) & =M \int \frac{\mathrm{d}^{3} \boldsymbol{\Delta}}{(2 \pi)^{3}}\left\{A_{\mathrm{NR}}(t)+\frac{\Delta^{2}}{8 M^{2} c^{2}}\left[A_{\mathrm{NR}}(t)+2 D_{\mathrm{NR}}(t)\right]\right\} e^{-i \boldsymbol{\Delta} \cdot \mathbf{r}} e^{-\frac{\sigma^{2}}{2} \boldsymbol{\Delta}^{2}}+\mathcal{O}\left(1 / c^{4}\right),
\end{aligned}
$$

\footnotetext{
${ }^{2}$ Since matrix elements are invariant under unitary transformations, unlike state kets or operators, it is more suitable to apply nonrelativistic reduction to matrix elements as a whole rather than to their individual components.
} 


$$
\rho_{\text {mass }}^{(j=1 / 2)}(\mathbf{r} ; \sigma)=M \int \frac{\mathrm{d}^{3} \boldsymbol{\Delta}}{(2 \pi)^{3}}\left\{A_{\mathrm{NR}}(t)+\frac{\boldsymbol{\Delta}^{2}}{4 M c^{2}}\left[A_{\mathrm{NR}}(t)+D_{\mathrm{NR}}(t)-2 J_{\mathrm{NR}}(t)\right]\right\} e^{-i \boldsymbol{\Delta} \cdot \mathbf{r}} e^{-\frac{\sigma^{2}}{2} \boldsymbol{\Delta}^{2}}+\mathcal{O}\left(1 / c^{4}\right) .
$$

This has exactly the expected form of a mass density plus a (nonrelativistic) kinetic energy density. Although we cannot take the $\sigma \rightarrow 0$ limit for the full (mass + kinetic) energy density at this order in $1 / c^{2}$, we can actually take this limit for the mass density by itself. This suggests that we can obtain a meaningful leading-order relativistic correction to the mass density. This suggestion must however be tempered by the realization that the separation of energy into mass and kinetic energy requires the ability to bring the system to rest, which is explicitly precluded by taking the $\sigma \rightarrow 0$ limit.

It is worthwhile to observe that the $\mathrm{NR}+\mathrm{LO}$ mass density for spin-half particles, as given in Eq. (35d), is identical in form to the Breit frame mass density given in Eq. (25b) (and previously found in Ref. [15] for instance). A caveat worth mentioning is that consistency of the nonrelativistic reduction requires expanding the form factors themselves in powers of $1 / c$, while the Breit frame density uses the exact relativistic form factors. Moreover, such a coincidence does not occur for spin-zero particles.

The procedure outlined here cannot be used at arbitrarily high orders in $1 / c$, and it is therefore not possible to define a fully relativistic 3D mass density through series of relativistic corrections. The dependence of $E_{\mathbf{p}}$ and $E_{\mathbf{p}^{\prime}}$ on $(\mathbf{P} \cdot \boldsymbol{\Delta})$ prevents factorizing the density integrand into a $\mathbf{P}$-dependent factor and $\boldsymbol{\Delta}$-dependent factor.

For both spin-zero and spin-half particles, the fully nonrelativistic $(c \rightarrow \infty)$ limit gives the same form for the mass density:

$$
\rho_{\mathrm{mass}}^{(\mathrm{FNR})}(\mathbf{r})=M \int \frac{\mathrm{d}^{3} \boldsymbol{\Delta}}{(2 \pi)^{3}} A_{\mathrm{NR}}(t) e^{-i \boldsymbol{\Delta} \cdot \mathbf{r}}
$$

This is spherically symmetric, and comparison to Eqs. (8) and (11) shows that this is related to the nonrelativistic limit of the fully relativistic $P^{+}$density:

$$
\rho_{\text {mass }}^{(\mathrm{FNR})}(r)=\lim _{c \rightarrow \infty} \frac{M}{P^{+}} \mathscr{A}\left[\rho_{P^{+}}^{(\mathrm{LF})}\left(b_{\perp}\right)\right](r)
$$

We thus see that the inverse Abel transform of the $P^{+}$ density does have a physical meaning, if it is accompanied by the $c \rightarrow \infty$ limit: it gives the fully nonrelativistic 3D mass density. Since the $c \rightarrow \infty$ limit is not invertible, this relation is not invertible either.

\section{Nonrelativistic stress tensor}

The $T^{i j}$ components of the EMT give the stress tensor. It is worth stressing that $T^{i j}$ does not only encode pressure and shear forces, but also contains contributions from the motion of the system. For instance, taking the FNR limit for a spin-zero system gives

$$
\begin{aligned}
& \left\langle\mathbf{p}^{\prime}, \mathbf{s}\left|T^{i j}(0)\right| \mathbf{p}, \mathbf{s}\right\rangle_{\mathrm{NR}}^{(j=0)} \\
& \quad=\frac{\mathbf{P}^{i} \mathbf{P}^{j}}{M} A_{\mathrm{NR}}(t)+\left(\frac{\boldsymbol{\Delta}^{i} \boldsymbol{\Delta}^{j}-\delta_{i j} \boldsymbol{\Delta}^{2}}{4 M}\right) D_{\mathrm{NR}}(t)+\mathcal{O}\left(1 / c^{2}\right) .
\end{aligned}
$$

As it is, the stress tensor cannot be used with Eq. (30) unless the $\sigma \rightarrow 0$ limit is avoided, because the factor $\mathbf{P}^{i} \mathbf{P}^{j}$ multiplying $A(t)$ will produce a $\sigma^{-2}$ divergence when $i=j$. However, we can define a density at nonzero $\sigma$,

$$
T_{\mathrm{NR}}^{i j}(\mathbf{r} ; \sigma)=\rho_{\mathrm{mass}}^{(\mathrm{NR})}(\mathbf{r} ; \sigma)\left\langle\mathbf{v}^{i} \mathbf{v}^{j}\right\rangle_{\sigma}+S_{\mathrm{NR}}^{i j}(\mathbf{r} ; \sigma),
$$

in the FNR, where

$$
\begin{aligned}
\left\langle\mathbf{v}^{i} \mathbf{v}^{j}\right\rangle_{\sigma} & =\int \frac{\mathrm{d}^{3} \mathbf{P}}{(2 \pi)^{3}} \frac{\mathbf{P}^{i} \mathbf{P}^{j}}{M^{2}}|\psi(\mathbf{P}, \mathbf{s} ; \sigma)|^{2}=\frac{\delta_{i j}}{M^{2} \sigma^{2}}, \\
S_{\mathrm{NR}}^{i j}(\mathbf{r} ; \sigma) & =\int \frac{\mathrm{d}^{3} \boldsymbol{\Delta}}{(2 \pi)^{3}}\left(\frac{\boldsymbol{\Delta}^{i} \boldsymbol{\Delta}^{j}-\delta_{i j} \boldsymbol{\Delta}^{2}}{4 M}\right) D_{\mathrm{NR}}(t) e^{-i \boldsymbol{\Delta} \cdot \mathbf{r}} e^{-\frac{\sigma^{2}}{2} \boldsymbol{\Delta}^{2}} .
\end{aligned}
$$

This has the form expected of the classical nonrelativistic stress tensor, with a piece encoding movement of the system and a piece expressing the comoving stress tensor $S^{i j}$. This is identical to the decomposition used to isolate $S_{\mathrm{LF}}^{i j}$ in the light front case in Ref. [17] and above. The comoving stress tensor is invariant under Galilean boosts, and thus can be interpreted as the stress tensor as seen by a comoving observer-a physical interpretation that is justified by having taken the fully nonrelativistic limit.

The comoving stress tensor is well defined in the $\sigma \rightarrow 0$ limit:

$S_{\mathrm{NR}}^{i j}(\mathbf{r})=\frac{1}{4 M}\left(\delta^{i j} \nabla^{2}-\nabla^{i} \nabla^{j}\right) \int \frac{\mathrm{d}^{3} \boldsymbol{\Delta}}{(2 \pi)^{3}} D_{\mathrm{NR}}(t) e^{-i \boldsymbol{\Delta} \cdot \mathbf{r}}$.

It is straightforward to show that $S_{\mathrm{NR}}^{i j}(\mathbf{r})$ also has this form for spin-half systems.

The leading relativistic corrections introduce factors of $\mathbf{P}^{2}$ and $(\mathbf{P} \cdot \boldsymbol{\Delta})^{2}$ into the quantity $S^{i j}$ itself. These preclude using the $\mathcal{O}\left(1 / c^{2}\right)$ corrections to define a density via Eq. (30). These factors are related to a breakdown of Galilean symmetry once relativistic effects are introduced. $S^{i j}$ is no longer invariant under boosts once relativistic corrections are introduced, so it no longer has a clear 
interpretation as being the comoving stress tensor, either. Whether it is possible to introduce relativistic corrections in a consistent way to the comoving stress tensor within the formalism we employ is unclear, so we proceed to employ only the nonrelativistic limit in this work.

As explained in Refs. [15,16], this comoving stress tensor can be decomposed into an isotropic pressure $p(r)$ and a pressure anisotropy $s(r)$ :

$$
S_{\mathrm{NR}}^{i j}(\mathbf{r})=\delta^{i j} p^{(\mathrm{NR})}(r)+\left(\frac{r^{i} r^{j}}{r^{2}}-\frac{1}{3} \delta^{i j}\right) s^{(\mathrm{NR})}(r) .
$$

By contracting this with unit vectors, it is possible to obtain directional pressures, e.g., radial and tangential pressures $[15,16]$ :

$$
\begin{aligned}
& p_{r}^{(\mathrm{NR})}(r)=\hat{r}_{i} \hat{r}_{j} S_{\mathrm{NR}}^{i j}(\mathbf{r})=p^{(\mathrm{NR})}(r)+\frac{2}{3} s^{(\mathrm{NR})}(r), \\
& p_{t}^{(\mathrm{NR})}(r)=\hat{\phi}_{i} \hat{\phi}_{j} S_{\mathrm{NR}}^{i j}(\mathbf{r})=p^{(\mathrm{NR})}(r)-\frac{1}{3} s^{(\mathrm{NR})}(r) .
\end{aligned}
$$

By integrating out the $z$ coordinate, one can obtain the 2D nonrelativistic stress tensor:

$$
S_{\mathrm{NR}}^{i j}\left(\mathbf{b}_{\perp}\right)=\frac{1}{4 M}\left(\delta^{i j} \nabla_{\perp}^{2}-\nabla_{\perp}^{i} \nabla_{\perp}^{j}\right) \int \frac{\mathrm{d}^{2} \boldsymbol{\Delta}_{\perp}}{(2 \pi)^{2}} D_{\mathrm{NR}}(t) e^{-i \boldsymbol{\Delta}_{\perp} \cdot \mathbf{b}_{\perp}},
$$

where dependence on $\mathbf{b}_{\perp}$ instead of $\mathbf{r}$ signifies that this is a 2D function. This can be compared to the light front comoving stress tensor in Eq. (12), giving

$$
S_{\mathrm{NR}}^{i j}\left(\mathbf{b}_{\perp}\right)=\lim _{c \rightarrow \infty} \frac{P^{+}}{M} S_{\mathrm{LF}}^{i j}\left(\mathbf{b}_{\perp}\right) .
$$

By comparing the 3D nonrelativistic eigenpressures in Eq. (42) to the 2D light front eigenpressures in Eq. (14), it is possible also to show that

$$
\begin{aligned}
& p_{t}^{(\mathrm{NR})}(r)=\lim _{c \rightarrow \infty} \frac{P^{+}}{M} \mathscr{A}^{-1}\left[p_{t}^{(\mathrm{LF})}\left(b_{\perp}\right)\right](r), \\
& p_{r}^{(\mathrm{NR})}(r)=\lim _{c \rightarrow \infty} \frac{2 P^{+}}{M} \mathscr{A}^{-1}\left[p_{r}^{(\mathrm{LF})}\left(b_{\perp}\right)\right](r) .
\end{aligned}
$$

These are compatible with the spin-half results of Ref. [30], although our result applies to spin-zero particles as well. Just as with the $P^{+}$density, we find that the inverse Abel transform has a physical meaning when accompanied by the $c \rightarrow \infty$ limit: it produces the 3D densities in the fully nonrelativistic limit.

\section{Nonrelativistic form factors}

The connection between the 3D nonrelativistic densities and the 2D light front densities, as given in Eqs. (37) and (45) are not invertible. This is so because the $c \rightarrow \infty$ limit cannot be undone. However, if the $c \rightarrow \infty$ limit had no effect on the form factors-i.e., if $A_{\mathrm{NR}}(t)=A(t)$ and $D_{\mathrm{NR}}(t)=D(t)$-then these relationships would actually be invertible. However, this is not the case, and we illustrate these points in the next section using a simple model.

\section{MODEL CALCULATIONS}

We will now illustrate the findings of this work with pedagogical model calculations, specifically using a generalization [25] of the $\phi^{3}$ model first used by Weinberg [33] and later by by Gunion et al. [34]. We use the interaction Lagrangian,

$$
\mathscr{L}_{I}[\Psi, \phi, \xi]=g \Psi(x) \phi(x) \xi(x),
$$

where all of the three different fields are spin-zero bosons. The $\Psi$ particle of mass $M$ represents the bound state of the two different constituents $\phi$ and $\xi$, of masses $m_{1}$ and $m_{2}$ respectively.

The pointlike coupling of this model is very simple, which is a pedagogic advantage, but short distance effects are emphasized [25] as the light-front wave function has a logarithmic divergence for small values of the transverse separation $b_{\perp}$ between the quarks. Furthermore, the asymptotic behavior of the electromagnetic form factor is $F(t) \sim \frac{\log ^{2}(-t)}{(-t)}$.

The electromagnetic current of the three-scalar model is given by

$$
\begin{aligned}
\left\langle p^{\prime}\left|j^{\mu}(0)\right| p\right\rangle= & 2 P^{\mu} F(t)=i g^{2} e_{1} \int \frac{\mathrm{d}^{4} k}{(2 \pi)^{4}} \frac{2 k^{\mu}}{\left[(k-P)^{2}-m_{2}^{2}\right]\left[(k+\Delta / 2)^{2}-m_{1}^{2}\right]\left[(k-\Delta / 2)^{2}-m_{1}^{2}\right]} \\
& +i g^{2} e_{2} \int \frac{\mathrm{d}^{4} k}{(2 \pi)^{4}} \frac{2 k^{\mu}}{\left[(k-P)^{2}-m_{1}^{2}\right]\left[(k+\Delta / 2)^{2}-m_{2}^{2}\right]\left[(k-\Delta / 2)^{2}-m_{2}^{2}\right]},
\end{aligned}
$$

where $P=\frac{1}{2}\left(p+p^{\prime}\right)$ and $\Delta=p^{\prime}-p$. This is a sum of contributions from the constituents with masses $m_{1}$ and $m_{2}$. It is important to emphasize here that the operator $j^{\mu}$ depends only on the fields $\phi(x)$ and $\xi(x)$, so there are not any direct contributions from the $\Psi(x)$ field. This is not the case for the EMT $T^{\mu \nu}$ : contributions from $-g^{\mu \nu} \mathscr{L}_{I}$ are present in this current. However, the $\Psi(x)$ field does not contribute to either $T^{++}$or to $T^{i j}$ when $i \neq j$, by virtue of these components of the metric vanishing. The ++ component of the gravitational current is given by 


$$
\begin{aligned}
\left\langle p^{\prime}\left|T^{++}(0)\right| p\right\rangle= & 2\left(P^{+}\right)^{2} A(t)=i g^{2} \int \frac{\mathrm{d}^{4} k}{(2 \pi)^{4}} \frac{2\left(k^{+}\right)^{2}}{\left[(k-P)^{2}-m_{2}^{2}\right]\left[(k+\Delta / 2)^{2}-m_{1}^{2}\right]\left[(k-\Delta / 2)^{2}-m_{1}^{2}\right]} \\
& +i g^{2} \int \frac{\mathrm{d}^{4} k}{(2 \pi)^{4}} \frac{2\left(k^{+}\right)^{2}}{\left[(k-P)^{2}-m_{1}^{2}\right]\left[(k+\Delta / 2)^{2}-m_{2}^{2}\right]\left[(k-\Delta / 2)^{2}-m_{2}^{2}\right]},
\end{aligned}
$$

and to isolate $D(t)$ we look at the 12 component:

$$
\begin{aligned}
\left\langle p^{\prime}\left|T^{12}(0)\right| p\right\rangle= & \frac{1}{2} \Delta^{1} \Delta^{2} D(t)=i g^{2} \int \frac{\mathrm{d}^{4} k}{(2 \pi)^{4}} \frac{\left(k_{1}-\Delta_{1} / 2\right)\left(k_{2}+\Delta_{2} / 2\right)+\left(k_{2}-\Delta_{2} / 2\right)\left(k_{1}+\Delta_{1} / 2\right)}{\left[(k-P)^{2}-m_{2}^{2}\right]\left[(k+\Delta / 2)^{2}-m_{1}^{2}\right]\left[(k-\Delta / 2)^{2}-m_{1}^{2}\right]} \\
& +i g^{2} \int \frac{\mathrm{d}^{4} k}{(2 \pi)^{4}} \frac{\left(k_{1}-\Delta_{1} / 2\right)\left(k_{2}+\Delta_{2} / 2\right)+\left(k_{2}-\Delta_{2} / 2\right)\left(k_{1}+\Delta_{1} / 2\right)}{\left[(k-P)^{2}-m_{1}^{2}\right]\left[(k+\Delta / 2)^{2}-m_{2}^{2}\right]\left[(k-\Delta / 2)^{2}-m_{2}^{2}\right]} .
\end{aligned}
$$

In particular, we shall consider examples with masses appropriate for a spin-less deuteron and a scalar pion. In that case, we take $m_{1}=m_{2}=m$. To simplify the notation, we additionally take $e_{1}+e_{2}=1$.

The integrals above can be evaluated using Feynman parameters. It is useful to start by considering the forward limit $\Delta^{\mu}=0$. Then,

$$
F(0)=\frac{g^{2}}{16 \pi^{2}} \int_{0}^{1} \mathrm{~d} x \frac{(1-x) x}{\mathcal{M}^{2}(x)},
$$

with

$$
\mathcal{M}^{2}(x) \equiv m^{2}-x(1-x) M^{2}
$$

The coupling constant $g$ is chosen to yield $F(0)=1$. An important consistency check that

$$
A(0)=F(0)
$$

is satisfied. We also find that

$$
D(0)=-\frac{g^{2}}{8 \pi^{2}} \int_{0}^{1} \mathrm{~d} x(1-x) \frac{1-(1-x)^{2} / 3}{\mathcal{M}^{2}(x)} .
$$

Defining the positive binding energy to be $B$ with $M=$ $2 m-B$ we find

$$
\begin{aligned}
& \lim _{B \rightarrow 0} D(0)=-\frac{11}{3}+\frac{32}{3 \pi} \sqrt{\frac{B}{2 M}}-\mathcal{O}\left(\frac{B}{M}\right) \\
& \lim _{B \rightarrow \infty} D(0)=-5+\frac{2}{15}\left(\frac{2 M}{M+B}\right)^{2}+\mathcal{O}\left(\frac{M^{4}}{B^{4}}\right)
\end{aligned}
$$

An equivalent procedure [25] to the use of Feynman parameters is to use the Drell-Yan frame, where $\Delta^{+}=0$, and integrate over $k^{-} \equiv k^{0}-k^{3}$. This enables one to obtain form factors in terms of light-front wave functions, and also simplifies taking the nonrelativistic limit. We also use the relative momentum $\boldsymbol{\kappa}=\mathbf{k}-x\left(\mathbf{P}-\boldsymbol{\Delta}_{\perp} / 2\right)$. The result is that

$$
\begin{aligned}
F(t)= & \frac{1}{2(2 \pi)^{3}} \int \mathrm{d}^{2} \boldsymbol{\kappa} \int_{0}^{1} \frac{\mathrm{d} x}{x(1-x)} \\
& \times \psi^{*}\left(x, \boldsymbol{\kappa}+(1-x) \boldsymbol{\Delta}_{\perp}\right) \psi(x, \boldsymbol{\kappa}),
\end{aligned}
$$

as found in Ref. [34], with the frame-independent light-front wave function $\psi(x, \boldsymbol{\kappa})$ given by

$$
\psi(x, \boldsymbol{\kappa}) \equiv g\left[M^{2}-\frac{\boldsymbol{\kappa}^{2}+m_{1}^{2}}{x}-\frac{\boldsymbol{\kappa}^{2}+m_{2}^{2}}{1-x}\right]^{-1},
$$

where $\kappa$ is the $\perp$-component of the transverse relative momentum, and $x$ is the fractional component of the longitudinal plus-component of the momentum carried by the constituent of mass $m_{1}$.

For equal mass particles we find

$$
A(t)=F(t)
$$

in this simple model, and identity that is very useful for deuteronlike kinematics, as we shall see below.

To aid in the calculations that follow, it is efficient to define a quantity:

$$
G(\alpha, \boldsymbol{\Delta}) \equiv \int \mathrm{d}^{2} \boldsymbol{\kappa} \psi^{*}\left(x, \boldsymbol{\kappa}+\alpha \boldsymbol{\Delta}_{\perp}\right) \psi(x, \boldsymbol{\kappa}),
$$

with $\alpha$ as either $x$ or $1-x$, depending on which particle is probed. Then (for instance)

$$
F(t)=\frac{1}{16 \pi^{3}} \int_{0}^{1} \frac{\mathrm{d} x}{x(1-x)} G\left(1-x, \Delta_{\perp}\right) .
$$

The use of Feynman parameters leads to the result:

$$
\begin{aligned}
G(\alpha, \boldsymbol{\Delta}) & =2 \pi g^{2} x^{2} \bar{x}^{2} \int_{0}^{1 / 2} \mathrm{~d} z \frac{1}{\mathcal{M}^{2}+\alpha^{2} Q^{2}\left(1 / 4-z^{2}\right)} \\
& =4 \pi g^{2} x^{2} \bar{x}^{2} \frac{\log \left(\frac{\sqrt{\alpha^{2} Q^{2}\left(4 \mathcal{M}^{2}+\alpha^{2} Q^{2}\right)}+2 \mathcal{M}^{2}-\alpha^{2} t}{2 \mathcal{M}^{2}}\right)}{\sqrt{\alpha^{2} Q^{2}\left(4 \mathcal{M}^{2}+\alpha^{2} Q^{2}\right)}} .
\end{aligned}
$$


Transverse densities are 2D Fourier transforms of the relevant form factors, such as

$$
\rho_{F}\left(b_{\perp}\right)=\int \frac{\mathrm{d}^{2} \boldsymbol{\Delta}_{\perp}}{(2 \pi)^{2}} e^{-i \boldsymbol{\Delta}_{\perp} \cdot \mathbf{b}_{\perp}} F(t),
$$

so that it is useful to introduce the coordinate-space wave function:

$$
\psi\left(x, \mathbf{b}_{\perp}\right)=\int \frac{\mathrm{d}^{2} \kappa}{(2 \pi)^{2}} \psi(x, \boldsymbol{\kappa}) e^{i \boldsymbol{k} \cdot \mathbf{b}_{\perp}} .
$$

In this model:

$$
\psi\left(x, b_{\perp}\right)=\frac{1}{(2 \pi)^{2}} \int \mathrm{d}^{2} \boldsymbol{\kappa} e^{i \boldsymbol{\kappa} \cdot \mathbf{b}} \psi(x, \boldsymbol{\kappa})=\frac{-g}{(2 \pi)} x \bar{x} K_{0}\left(\mathcal{M} b_{\perp}\right),
$$

where $K_{0}(x)$ is a modified Bessel function of the second kind, and where $\bar{x} \equiv 1-x$. In terms of this wave function, the density corresponding to the electromagnetic form factor $\rho_{F}$ is given by

$$
\rho_{F}\left(b_{\perp}\right)=\int_{0}^{1} \frac{\mathrm{d} x}{(1-x)^{2}}\left|\psi\left(x, \frac{b_{\perp}}{1-x}\right)\right|^{2} .
$$

Similarly, one may show that

$$
\frac{1}{2} \Delta_{1} \Delta_{2} D(t)=\int \frac{\mathrm{d}^{2} \boldsymbol{\kappa}}{(2 \pi)^{3}} \int_{0}^{1} \frac{\mathrm{d} x}{x(1-x)}\left(2 \kappa_{1} \kappa_{2}-\Delta_{1} \Delta_{2} / 2\right)\left[\frac{1}{x} \psi^{*}\left(x, \boldsymbol{\kappa}+(1-x) \boldsymbol{\Delta}_{\perp} / 2\right) \psi\left(x, \boldsymbol{\kappa}-(1-x) \boldsymbol{\Delta}_{\perp} / 2\right)\right] .
$$

Evaluation of the term proportional to $\kappa_{1} \kappa_{2}$ must result in a term proportional to $\Delta_{1} \Delta_{2}$. However, it is worthwhile to find an explicit expression for $D(t)$. This may be done by expressing $D(t)$ in terms of the coordinate-space wave function, $\psi\left(x, \mathbf{b}_{\perp}\right)$. Some algebra leads to the expression

$$
D(t)=-4 \int_{0}^{1} \frac{\mathrm{d} x}{x(1-x)^{2}} \int \mathrm{d} b_{\perp} b_{\perp}\left|\psi^{\prime}\left(x, b_{\perp}\right)\right|^{2} \frac{J_{2}\left(x\left|\boldsymbol{\Delta}_{\perp}\right| b_{\perp}\right)}{(-t)}-\int \frac{\mathrm{d} x(1+x)}{x(1-x)} \int \mathrm{d} b_{\perp} b_{\perp}\left|\psi\left(x, b_{\perp}\right)\right|^{2} J_{0}\left(x\left|\boldsymbol{\Delta}_{\perp}\right| b_{\perp}\right),
$$

where $\psi^{\prime}\left(x, b_{\perp}\right) \equiv \frac{\partial \psi(x, b)}{\partial b}$. The quadrupole nature of $D(t)$ is exhibited by the appearance of the Bessel function of order 2 in Eq. (66).

The transverse density is obtained in the same way. For simplicity, we examine the light front version of Polyakov's stress potential function $\tilde{D}\left(b_{\perp}\right)$ defined in Eq. (15). for which we find

$$
4 P^{+} \tilde{D}\left(b_{\perp}\right)=\frac{-1}{\pi} \int \frac{\mathrm{d} x}{x(1-x)}\left[f\left(x, \frac{b_{\perp}}{\bar{x}}\right)+\frac{1}{2}\left(\frac{1-x^{2}}{x^{2} \bar{x}}\left|\psi\left(x, \frac{b_{\perp}}{x}\right)\right|^{2}\right)\right]
$$

with

$$
f(\alpha, b) \equiv \int_{b / \alpha}^{\infty} \mathrm{d} b_{\perp}^{\prime} b_{\perp}^{\prime}\left(1-\frac{b^{2}}{\alpha^{2} b^{\prime 2}}\right)\left|\psi^{\prime}\left(\alpha, b^{\prime}\right)\right|^{2}
$$

The above derivation of $D(t)$ exhibits the dependence on the wave function and is thus useful in obtaining the nonrelativistic limit. One may instead proceed more directly to determine $D(t)$ by starting with Eq. (65) and using Feynman parameters to obtain

$$
D(t)=\frac{-1}{8 \pi^{3}} \int_{0}^{1} \mathrm{~d} x \frac{1}{x^{2} \bar{x}} \tilde{G}(\bar{x}, t)
$$

with

$$
\tilde{G}(\alpha, t) \equiv 2 \pi g^{2} x^{2} \bar{x}^{2} \int_{0}^{1} \mathrm{~d} y \frac{\left(1-y^{2} \alpha^{2}\right)}{\mathcal{M}^{2}-\alpha^{2} t / 4\left(1-y^{2}\right)}=8 \pi g^{2} x^{2} \bar{x}^{2} \frac{\left|\boldsymbol{\Delta}_{\perp}\right|-\frac{\left(4-\left(\alpha^{2}-1\right) t\right) \tanh ^{-1}\left(\frac{\alpha\left|\Delta_{\perp}\right|}{\sqrt{4-\alpha^{2} t}}\right)}{\alpha \sqrt{4-\alpha^{2} t}}}{\left|\boldsymbol{\Delta}_{\perp}\right|^{3}}
$$

\section{A. Breit-frame densities and transverse pseudodensities}

The three-dimensional Breit-frame densities are given in Sec. III B above. From these, one obtains the 2D Breit frame densities through an Abel transform. For spin-zero hadrons specifically, one has 


$$
\tilde{D}_{\mathrm{BF}}\left(b_{\perp}\right)=\int_{-\infty}^{\infty} \mathrm{d} z \tilde{D}_{\mathrm{BF}}(r)=\int \frac{\mathrm{d}^{2} \boldsymbol{\Delta}_{\perp}}{(2 \pi)^{2}} \frac{D(t)}{\sqrt{1-t /\left(4 M^{2}\right)}} e^{-i \boldsymbol{\Delta}_{\perp} \cdot \mathbf{b}_{\perp}},
$$

for example. If $M \rightarrow \infty$ then $\tilde{D}_{\mathrm{BF}}\left(b_{\perp}\right) \rightarrow \tilde{D}\left(b_{\perp}\right)$, with the latter being the relativistically exact (light front) density. Since the integral goes over all values of $t$, however, the equality does not hold. At best, one could have $\tilde{D}_{\mathrm{BF}}\left(b_{\perp}\right) \approx$ $\tilde{D}\left(b_{\perp}\right)$ if $D\left(-t \rightarrow 4 M^{2}\right) \approx 0$. Therefore we refer to $\tilde{D}_{\mathrm{BF}}\left(b_{\perp}\right)$ as a transverse pseudodensity.

The integral appearing in Eq. (70) provides a numerical challenge because the asymptotic limit is $D(t) \sim \log ^{2}(-t) /$ $(-t)$. This means that an expansion in powers of $-t^{2} / 4 M^{2}$ diverges. A valid numerical procedure is obtained by relating $\tilde{D}_{\mathrm{BF}}\left(b_{\perp}\right)$ to the relativistic transverse density $\tilde{D}\left(b_{\perp}\right)$. This is achieved by using Eq. (15) and the relation

$$
\int \frac{\mathrm{d}^{2} \boldsymbol{\Delta}_{\perp}}{\left(2 \pi^{2}\right)} \frac{e^{i \boldsymbol{\Delta}_{\perp} \cdot \mathbf{s}}}{\sqrt{1-t^{2} /\left(4 M^{2}\right)}}=\frac{1}{\pi} \frac{e^{-2 M s}}{s}
$$

so that

$$
\tilde{D}_{\mathrm{BF}}\left(b_{\perp}\right)=\frac{M}{\pi} \int \mathrm{d}^{2} s \tilde{D}\left(\left|\mathbf{b}_{\perp}-\mathbf{s}\right|\right) \frac{e^{-2 M s}}{s} .
$$

This expression is amenable to two-dimensional numerical integration.

\section{B. Nonrelativistic limit}

The conventional lore is that the electromagnetic form factor is the three-dimensional Fourier transform (3DFT) of the charge density. This idea emerges only by taking the fully nonrelativistic limit.

We briefly review [25] how the 3DFT emerges. Our starting point is the wave function Eq. (56) and the form factor Eq. (55). Recall that the quantity $x=k^{+} / P^{+}$. In the nonrelativistic limit the energy $k^{0}=m_{1}$ and $k^{+}=m_{1}+\kappa^{3}$, where $\kappa^{3}$ is the third component of the relative longitudinal momentum. Further we define the positive binding energy $B$ so that

$$
M \equiv m_{1}+m_{2}-B
$$

Then $[51,52]$

$x=\frac{m_{1}+\kappa^{3}}{M}, \quad 1-x=\frac{M-m_{1}-\kappa^{3}}{M}=\frac{m_{2}-B-\kappa^{3}}{M}$.

To obtain the nonrelativistic wave function we express the denominator appearing in Eq. (56) in terms of $\kappa^{3}$. This gives

$$
M^{2}-\frac{\kappa^{2}+m_{1}^{2}}{x}-\frac{\kappa^{2}+m_{2}^{2}}{1-x} \approx 2 M\left(-B-\frac{\kappa^{2}}{2 \mu}\right)
$$

where

$\kappa^{2} \equiv \boldsymbol{\kappa}^{2}+\kappa_{3}^{2}, \quad \boldsymbol{\kappa}=\boldsymbol{\kappa}+\kappa^{3} \hat{\mathbf{z}}, \quad \mu \equiv \frac{m_{1} m_{2}}{m_{1}+m_{2}}$

In deriving Eq. (75), we have dropped terms of order $(v / c)^{3}=(k / m)^{3}$ and higher, and terms of order $B / M$ and higher. The result is that Eq. (75) is recognizable as $2 M$ times the inverse of the nonrelativistic propagator.

The next step is to determine the coordinate form of the nonrelativistic wave function $\psi_{\mathrm{NR}}(\mathbf{r})$ (where $\mathbf{r}$ is canonically conjugate to $\kappa$ ) and to show that the nonrelativistic form factor is a three-dimensional Fourier transform of $\left|\psi_{\mathrm{NR}}(\mathbf{r})\right|^{2}$. First use the nonrelativistic approximation Eq. (75) in Eq. (56) to find

$$
\psi_{\mathrm{NR}}(\boldsymbol{\kappa})=\frac{-\mu g}{M\left(\kappa^{2}+\lambda^{2}\right)}, \quad \lambda^{2} \equiv 2 \mu B
$$

The coordinate-space wave function $\psi_{\mathrm{NR}}(\mathbf{r})$ is given by

$\psi_{\mathrm{NR}}(\mathbf{r})=\frac{1}{(2 \pi)^{3 / 2}} \int \mathrm{d}^{3} \boldsymbol{\kappa} e^{i \boldsymbol{\kappa} \cdot \boldsymbol{r}} \psi_{\mathrm{NR}}(\boldsymbol{\kappa})=-\frac{\mu g}{2 M} \sqrt{\frac{\pi}{2}} \frac{e^{-\lambda r}}{r}$.

The expression Eq. (78) is seen as the standard result obtained for the bound state of a two-particle system interacting via an attractive delta function potential. It is also the effective range approximation [53], now known as the leading-order term in effective field theory [54].

The wave functions Eqs. (77) and (78) enable us to examine the conditions needed for the approximations Eq. (74) to be valid. For Eq. (74) to work, we need $\kappa^{2} \ll m_{1,2}^{2}$. The wave functions include all corrections to masses of order $\kappa^{2} / m_{1,2}^{2}$, and therefore no further corrections of order $\kappa / m_{1,2}$ or $B / m_{1,2}$ should be included. Thus in evaluating the form factor we should use

$$
\begin{aligned}
\mathrm{d} x & \rightarrow \frac{\mathrm{d} \boldsymbol{\kappa}^{3}}{M}, \\
x(1-x) & \rightarrow \frac{\mu}{M}, \\
(1-x) \boldsymbol{\Delta} & \rightarrow \frac{m_{2}}{M} \boldsymbol{\Delta} .
\end{aligned}
$$


The nonrelativistic electromagnetic form factor $F_{\mathrm{NR}}(t)$ is obtained by using Eq. (77) in the expression for the form factor Eq. (55), and taking the nonrelativistic limit as defined above. The result is

$$
F_{\mathrm{NR}}(t)=\frac{1}{2(2 \pi)^{3} \mu} \int \mathrm{d}^{3} \mathbf{r}\left|\psi_{\mathrm{NR}}(\mathbf{r})\right|^{2} e^{-i \boldsymbol{\Delta} \cdot \mathbf{r}^{\frac{m_{2}}{M}}}
$$

This conforms to the commonplace expectation that the form factor is a three-dimensional Fourier transform of the density. One may extract the density $\left|\psi_{\mathrm{NR}}\left(b_{\perp}\right)\right|^{2}$ by taking the Fourier transform of the form factor:

$$
\int \mathrm{d}^{3} \boldsymbol{\Delta} F_{\mathrm{NR}}(t) e^{i \boldsymbol{\Delta} \cdot \mathbf{R}}=\frac{1}{2 \mu}\left|\psi_{\mathrm{NR}}\left(\frac{M}{m_{2}} R\right)\right|^{2}
$$

Similarly, the nonrelativistic gravitational form factor is given by

$$
A_{\mathrm{NR}}(t)=\frac{1}{2(2 \pi)^{3} \mu} \int \mathrm{d}^{3} \mathbf{r}\left|\psi_{\mathrm{NR}}(\mathbf{r})\right|^{2}\left[m_{1} e^{-i \boldsymbol{\Delta} \cdot \mathbf{r} \frac{m_{2}}{m_{1}+m_{2}}}+m_{2} e^{i \Delta \cdot \mathbf{r} \frac{m_{1}}{m_{1}+m_{2}}}\right]
$$

The principle difference between the relativistic and nonrelativistic computations of form factors occurs e.g., in Eq. (79c): the variable factor $(1-x)$ is replaced by a constant.

We may evaluate the integrals immediately to find

$$
\begin{gathered}
F_{\mathrm{NR}}(t)=\frac{\tan ^{-1} \frac{\left|\boldsymbol{\Delta}_{\perp}\right| m_{2}}{2\left(m_{1}+m_{2}\right) \lambda}}{\frac{\left|\boldsymbol{\Delta}_{\perp}\right| m_{2}}{2\left(m_{1}+m_{2}\right) \lambda}}, \\
A_{\mathrm{NR}}(t)=\frac{2 \lambda}{\left|\boldsymbol{\Delta}_{\perp}\right|}\left[\frac{m_{1}}{m_{2}} \tan ^{-1}\left(\frac{\left|\boldsymbol{\Delta}_{\perp}\right| m_{2}}{2\left(m_{1}+m_{2}\right) \lambda}\right)+\frac{m_{2}}{m_{1}} \tan ^{-1}\left(\frac{\left|\boldsymbol{\Delta}_{\perp}\right| m_{1}}{2\left(m_{1}+m_{2}\right) \lambda}\right)\right],
\end{gathered}
$$

where the coupling constants and other constants enter in such a manner as to make $F_{\mathrm{NR}}(0)=1$. Note that if $m_{1}=m_{2}$ one has $A_{\mathrm{NR}}(t)=F_{\mathrm{NR}}(t)$, as noted previously of the fully relativistic case.

It is easiest to get $D_{\mathrm{NR}}$ using momentum space techniques First choose $g$ such that $F_{\mathrm{NR}}(0)=1$, which leads to

$$
g^{2}=\frac{16 \pi M^{2} \lambda}{\mu}
$$

Then take the nonrelativistic limit of Eq. (65), defining $\alpha \equiv m / M$. We obtain

$$
\begin{aligned}
\Delta_{1} \Delta_{2} D_{\mathrm{NR}}(t) & =\Delta_{1} \Delta_{2} \frac{1}{2 \pi^{3} \mu} \int \mathrm{d}^{3} \boldsymbol{\kappa}\left(2 \kappa_{1} \kappa_{2}-\Delta_{1} \Delta_{2} / 2\right) \psi_{\mathrm{NR}}^{*}(\boldsymbol{\kappa}+\alpha \boldsymbol{\Delta} / 2) \psi_{\mathrm{NR}}(\boldsymbol{\kappa}-\alpha \boldsymbol{\Delta} / 2) \\
& =8 \lambda / \pi^{2} \int \mathrm{d}^{3} \boldsymbol{\kappa}\left(2 \alpha^{2} z^{2} \Delta_{1} \Delta_{2}-\Delta_{1} \Delta_{2} / 2\right) \int_{-1 / 2}^{1 / 2} \mathrm{~d} z \frac{1}{\left(\kappa^{2}+\lambda^{2}+\alpha^{2} \Delta^{2}\left(1 / 4-z^{2}\right)\right)^{2}} \\
D_{\mathrm{NR}}(t) & =-8 \lambda \int_{0}^{1 / 2} \mathrm{~d} z \frac{\left(1-4 \alpha^{2} z^{2}\right)}{\sqrt{\lambda^{2}-t\left(1 / 4-z^{2}\right)}} \\
& =8 \lambda\left(\frac{\lambda}{t}-\frac{\left(4 \lambda^{2}-\left(\alpha^{2}-2\right) t\right) \csc ^{-1}\left(\sqrt{1-\frac{4 \lambda^{2}}{\alpha^{2} t}}\right)}{2 \alpha|\Delta|^{3}}\right)
\end{aligned}
$$

The value at zero momentum transfer is of interest, and it can be obtained immediately from Eq. (85c) to be

$$
D_{\mathrm{NR}}(0)=-4\left(1-\frac{m^{2}}{3 M^{2}}\right)=-4\left(1-\frac{1}{12}\left(1+\frac{B}{M}\right)^{2}\right)
$$



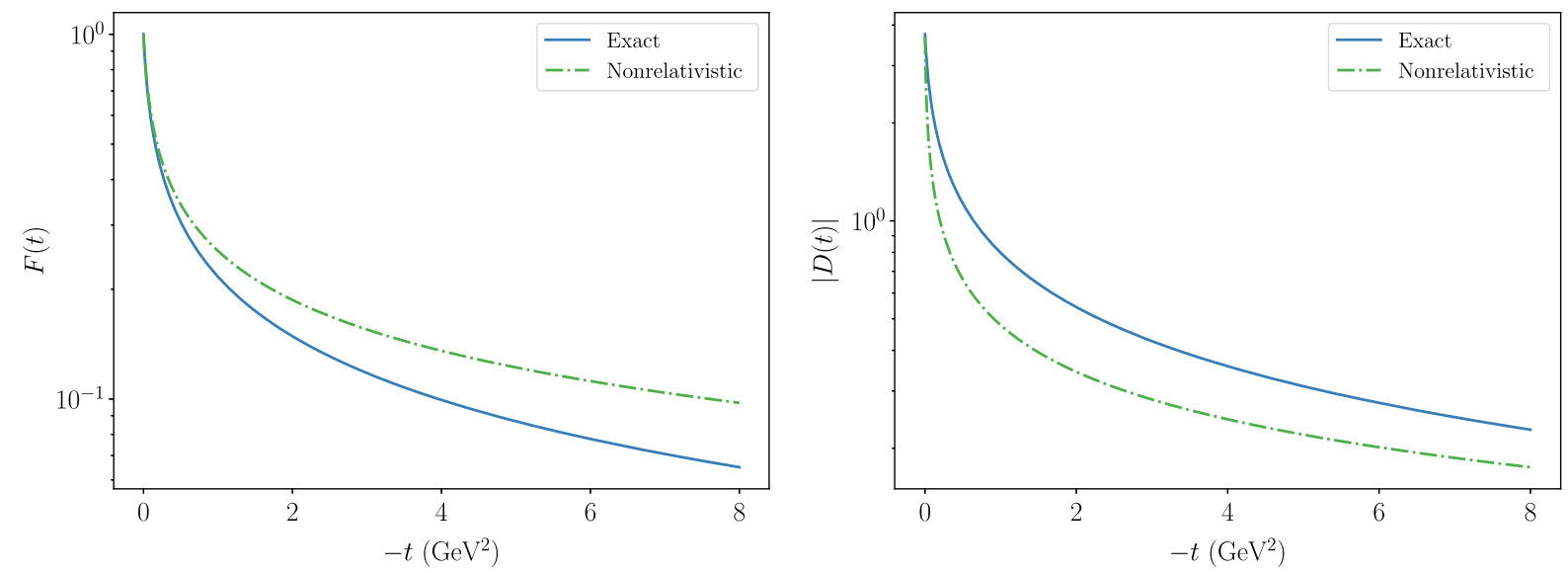

FIG. 2. Form factors for a scalar toy model with deuteronlike kinematics. Exact results for (left panel) $F(t)$ and (right panel) $D(t)$ are compared to their nonrelativistic approximations.

For weak binding (with $m \approx M / 2$ ), we find $D_{\mathrm{NR}}(0)=$ $-11 / 3$, which agrees with the fully relativistic result in this limit. For strong binding $(B \approx M)$, we find $D_{\mathrm{NR}}(0)=-8 / 3$. For very strong binding with $B / M>2 \sqrt{3}-1 \approx 2.5$, we find $D_{\mathrm{NR}}(0)>0$, in violation of Polyakov's negativity condition. This suggests that the nonrelativistic model is invalid for such large binding energies.

To evaluate the nonrelativistic transverse stress potential $\tilde{D}_{\mathrm{NR}}\left(b_{\perp}\right)$, we use the identity

$$
\begin{gathered}
\int \frac{\mathrm{d}^{2} \boldsymbol{\Delta}_{\perp}}{(2 \pi)^{2}} e^{i \boldsymbol{\Delta}_{\perp} \cdot \mathbf{b}_{\perp}} \frac{1}{\sqrt{\lambda^{2}-t\left(1 / 4-z^{2}\right)}} \\
=\frac{1}{2 \pi b_{\perp} \sqrt{\left(1 / 4-z^{2}\right)}} e^{\frac{-b_{\perp} \lambda}{\sqrt{1 / 4-z^{2}}}},
\end{gathered}
$$

giving us (with $\alpha=1 / 2$ )

$4 P^{+} \tilde{D}_{\mathrm{NR}}\left(b_{\perp}\right)=-\frac{8 \lambda}{2 \pi b_{\perp}} \int_{0}^{1 / 2} \mathrm{~d} z \frac{1-z^{2}}{\sqrt{1 / 4-z^{2}}} e^{\frac{-b_{\perp} \lambda}{\sqrt{1 / 4-z^{2}}}}$.

\section{Selected examples}

We examine two specific examples, with weak and strong binding respectively. The former is appropriate for deuteronlike kinematics and the latter for pionlike kinematics, especially the kinematics in the light front model of Ref. [55].

\section{Deuteronlike kinematics}

We first look at an example with weak binding, namely deuteronlike kinematics with $M=1.875 \mathrm{GeV}$ and $B=0.001 M$. Recall that $M=2 m-B$, with $B>0$.

In Fig. 2, we compare the exact relativistic electromagnetic form factor $F(t)$ and exact stress form factor $D(t)$ to their nonrelativistic approximations $F_{\mathrm{NR}}(t)$ and $D_{\mathrm{NR}}(t)$ as functions of $-t$. The derivation of the exact and nonrelativistic form factors serves as a rough guide for the significance of relativistic effects in the system.

The exact and nonrelativistic $F(t)$ are close when $-t / M^{2}<0.1$ (or $-t \approx 0.4 \mathrm{GeV}^{2}$ ), but diverge significantly at moderate and larger $-t$. Since $D(0)$ is not protected by a conservation law [unlike $F(0)$ or $A(0)$ ], it is possible for the exact and nonrelativistic values to differ. Indeed we find an exact $D$-term value of $D(0)=-3.74$ and a nonrelativistic value of $D_{\mathrm{NR}}(0)=-3.67$, which are fairly close in magnitude, but non-negligible. The differences between the exact and nonrelativistic form factors illustrate the differences discussed in Eq. (33) and related paragraphs.

Next, we compare the densities entailed by the relativistic and nonrelativistic $D(t)$ in Fig. 3. In particular, the direct Fourier transform $\tilde{D}\left(b_{\perp}\right)$ [as defined in Eq. (15)] and the radial pressure are both examined. For spin-zero targets, the Breit frame pseudodensity additionally differs from both the exact light front result due to the appearance of the factor $1 / \sqrt{1-t / 4 M^{2}}$, so it is compared to the exact and nonrelativistic results in both cases.

In the left panel of Fig. 3, the areas under the exact and Breit frame curves are the same, since the two-dimensional integrals of Eqs. (15) and (70) are equal. However, the factor $1 / \sqrt{1-t / 4 M^{2}}$ in Eq. (70) leads to the suppression of $\tilde{D}_{\mathrm{BF}}\left(b_{\perp}\right)$ at small values of $b_{\perp}$ and enhancement at moderate values, both by small amounts. At large values, the densities become equal, showing the corrections related to the factor $1 / \sqrt{1-t / 4 M^{2}}$ become negligible at large distances. However, the nonrelativistic result for $\tilde{D}\left(b_{\perp}\right)$ is significantly different from the exact and Breit frame results, suggesting that relativistic effects may persist in mechanical densities even at fairly large distances.

This same trend can be seen in the radial pressure, as depicted in the right panel of Fig. 3. Relativistic effects propagate to large $b_{\perp}$, even when they would be expected to vanish. This occurs because the pressure does not correspond to a conserved current, and is thus sensitive 

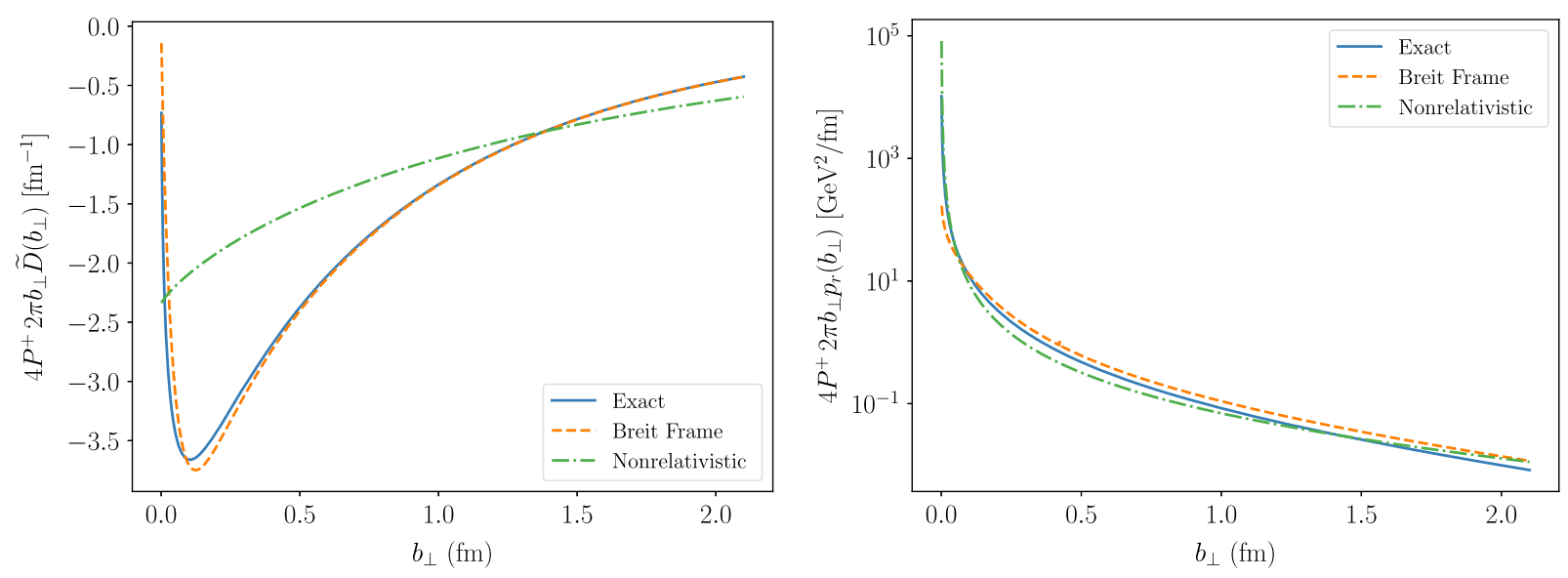

FIG. 3. D-term related densities for a scalar toy model with deuteronlike kinematics. Exact results, Breit frame results, and nonrelativistic approximations for (left panel) the potential $\tilde{D}\left(b_{\perp}\right)$ and (right panel) the 2D radial pressure $p_{r}\left(b_{\perp}\right)$ are compared.

to the details of the dynamics of a system (see Refs. [56,57] for examples of cases where the details of dynamics are significant). The details of the dynamics affect the overall mechanical structure of the hadron, and not just local aspects of the structure at small distances. Note that the log scale in the right panel of Fig. 3 covers more than 6 orders of magnitude, so that the apparently small differences are actually rather large.

For a slightly more detailed perspective, consider the right-hand side of Eq. (65), which was used in obtaining the relativistic $\tilde{D}\left(b_{\perp}\right)$. The matrix element is weighted by a factor $\frac{1}{x}$ compared to the matrix elements for the densities associated with $F(t)$ and $A(t)$. This factor increases the integrand when $x \sim 0$ or $x \sim 1$, conditions that are explicitly discounted by the nonrelativistic limit in which (for equal mass constituents) we make the replacement $\frac{1}{x} \mapsto \frac{1}{\alpha} \approx 2$.

The overall lesson of this case is that, because the mechanical properties of a hadron are not protected by a conservation law, they are sensitive to the details of dynamics, and accordingly nonrelativistic effects can have

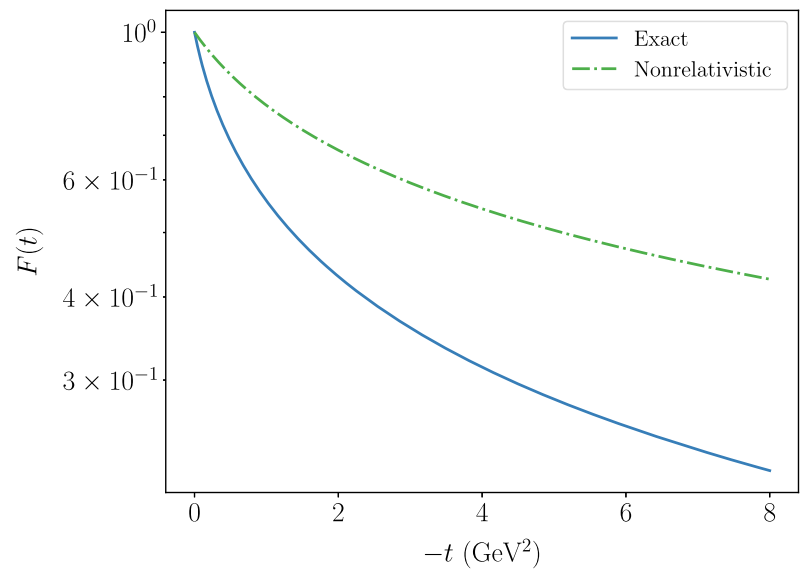

a significant effect even at large distances and even for weakly bound systems.

\section{Pionlike kinematics}

We now consider the scalar toy model with kinematics appropriate for a constituent quark model of the pion. We use a "quark" mass of $210 \mathrm{MeV}$, which leads to an excellent description of the pion's electromagnetic form factor [55]. With $M=140 \mathrm{MeV}$, this means that $B / M=2$, and according to Eq. (86) we get $D_{\mathrm{NR}}(0)=-1$, a result that immediately demonstrates the importance of relativistic effects for this model.

In Fig. 4, we present the exact and nonrelativistic form factors $F(t)$ and $D(t)$. As expected, the relativistic effects are substantial. Even at $t=0$, we have $D(0)=-4.9$ and $D_{\mathrm{NR}}(0)=-1$, about a fifth of the relativistically exact value. Moreover, the nonrelativistic approximation of $D(t)$ has a zero crossing that is absent in the exact result, as seen in the right panel of Fig. 4. This is because of the increasing importance of $z$ values near $\frac{1}{2}$ with larger $\Delta^{2}$, which causes

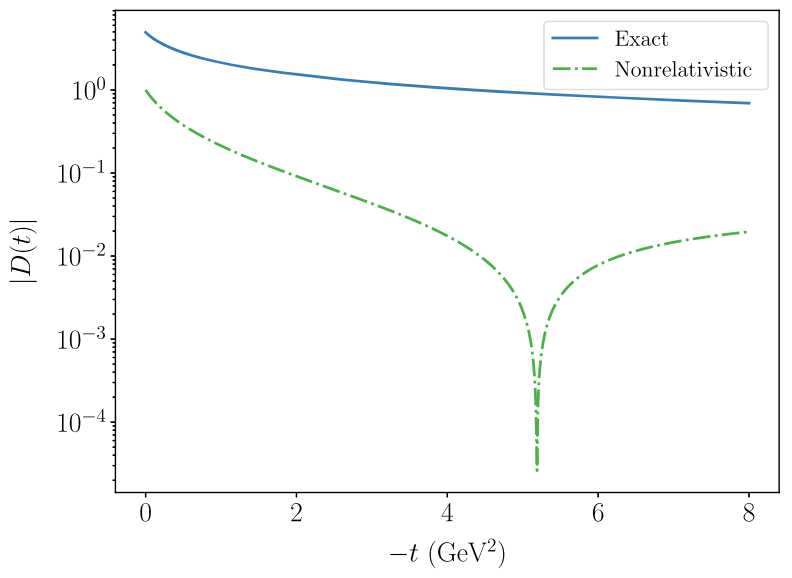

FIG. 4. Form factors for a scalar toy model with pionlike kinematics. Exact results for (left panel) $F(t)$ and (right panel) $D(t)$ are compared to their nonrelativistic approximations. 

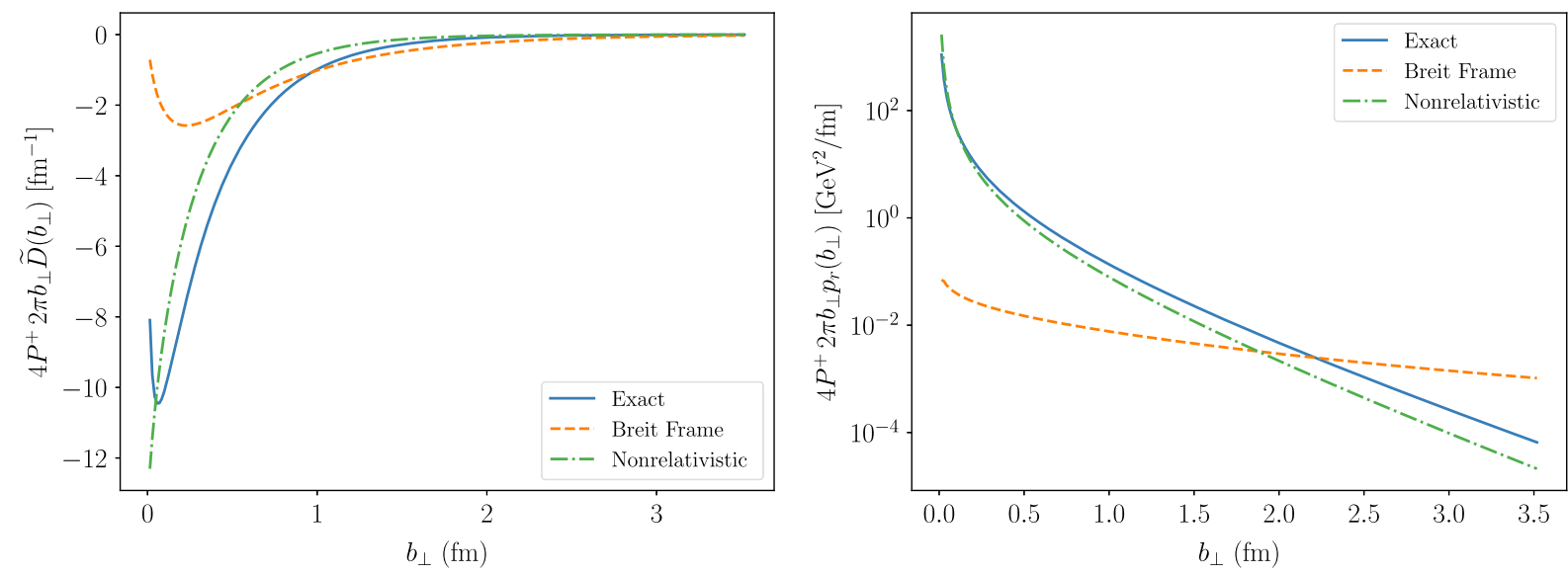

FIG. 5. D-term related densities for a scalar toy model with pionlike kinematics. Exact results, Breit frame results, and nonrelativistic approximations for (left panel) the potential $\tilde{D}\left(b_{\perp}\right)$ and (right panel) the $2 \mathrm{D}$ radial pressure $p_{r}\left(b_{\perp}\right)$ are compared.

the second term in the integrand of Eq. (85c) to dominate over the first term.

The density $\tilde{D}\left(b_{\perp}\right)$ and radial pressure are shown in Fig. 5. As expected, there are very substantial differences between the relativistic light front density, Breit frame pseudodensity, and nonrelativistic approximation. Remarkably, the Breit frame result is a worse approximation to the relativistically exact light front density in this case than the nonrelativistic approximation. This demonstrates the significance of the extraneous factor $1 / \sqrt{1-t /\left(4 M^{2}\right)}$ present in the Breit frame densities, and strongly forces us to the conclusion that the findings of Refs. [30,31] cannot be applied outside of the spin-half case, where those results hold only by accident.

\section{SUMMARY AND CONCLUSIONS}

In this work, we obtained exact relativistic (light front) and approximate nonrelativistic expressions for densities in spin-zero in spin-half hadrons. Focus was placed on the $P^{+}$ and mass densities, as well as the pressures encoded by the stress tensor as seen from the perspective of an observer comoving with the hadron. We compared the exact and nonrelativistic expressions to those obtained in the Breit frame formalism. We find that, in general, the Breit frame densities do not have a direct correspondence with the exact light front densities, even through Abel transforms. This failure of correspondence occurs in the spin-zero case because of an extraneous factor $1 / \sqrt{1-t /\left(4 M^{2}\right)}$ present in the integrand of every spin-zero Breit frame density, and in both cases because the relativistic light front densities do not exhibit spherical symmetry. The latter of these facts is illustrated for spin-half hadrons in particular by the azimuthal dependence of $P^{+}$densities and pressures of transversely polarized states, to which the inverse Abel transform is inapplicable even formally. In general, however, the light front formalism lacks spherical symmetry, since there is no $\mathrm{SO}(3)$ subgroup of the Poincare group that commutes with $P^{-}$[32].
The significance of both relativistic effects and the extraneous term $1 / \sqrt{1-t /\left(4 M^{2}\right)}$ present in the spin-zero Breit frame densities is illustrated through a pedagogical model. Relativistic effects were found to affect the mechanical structure of a composite system significantly, even for weakly bound systems and at large distances-in contrast to the electromagnetic density or mass density, both of which are protected by conservation laws. Moreover, for strongly bound systems, we found that the extraneous factor $1 / \sqrt{1-t /\left(4 M^{2}\right)}$ makes the (Abel transform of the) Breit frame density a poor approximation to the relativistic light front density.

Taking the inverse Abel transform of a transverse light front density can, at best, return a partially nonrelativistic approximation. This approximation is partially nonrelativistic, since a nonrelativistic approximation of the internal dynamics has not been applied to the form factors associated with the density, but instead only to the accompanying Lorentz tensors. In some circumstances, such as neutron star structure, this approximation may be warranted (cf. Ref. [36] for an example of this application), but one should bear in mind that this operation is an approximation that eliminates effects due to boosts from the target's rest frame. For targets with wave functions localized to a smaller distance than their reduced Compton wavelength, or targets for which a finer resolution of internal structure than the Compton wavelength is desired-such as hadrons-this approximation cannot be justified.

\section{ACKNOWLEDGMENTS}

We would like to thank Matthias Burkardt, Wim Cosyn, Xiangdong $\mathrm{Ji}$, and Simonetta Liuti for illuminating discussions on the topics covered in this paper. This work was supported by the U.S. Department of Energy Office of Science, Office of Nuclear Physics under Award No. DEFG02-97ER-41014. 
[1] I. Y. Kobzarev and L. B. Okun, Zh. Eksp. Teor. Fiz. 43, 1904 (1962).

[2] X.-D. Ji, Phys. Rev. Lett. 74, 1071 (1995).

[3] X.-D. Ji, Phys. Rev. D 52, 271 (1995).

[4] C. Lorcé, Eur. Phys. J. C 78, 120 (2018).

[5] Y. Hatta, A. Rajan, and K. Tanaka, J. High Energy Phys. 12 (2018) 008.

[6] A. Metz, B. Pasquini, and S. Rodini, Phys. Rev. D 102, 114042 (2020).

[7] X. Ji, Front. Phys. 16, 64601 (2021).

[8] C. Lorcé, A. Metz, B. Pasquini, and S. Rodini, J. High Energy Phys. 11 (2021) 121.

[9] J. Ashman et al. (European Muon Collaboration), Phys. Lett. B 206, 364 (1988).

[10] X.-D. Ji, Phys. Rev. Lett. 78, 610 (1997).

[11] E. Leader and C. Lorcé, Phys. Rep. 541, 163 (2014).

[12] M. Wakamatsu, Int. J. Mod. Phys. A 29, 1430012 (2014).

[13] X. Ji, F. Yuan, and Y. Zhao, Nat. Rev. Phys. 3, 27 (2021).

[14] M. V. Polyakov, Phys. Lett. B 555, 57 (2003).

[15] M. V. Polyakov and P. Schweitzer, Int. J. Mod. Phys. A 33, 1830025 (2018).

[16] C. Lorcé, H. Moutarde, and A. P. Trawiński, Eur. Phys. J. C 79, 89 (2019).

[17] A. Freese and G. A. Miller, Phys. Rev. D 103, 094023 (2021).

[18] V. D. Burkert, L. Elouadrhiri, and F. X. Girod, Nature (London) 557, 396 (2018).

[19] H. Dutrieux, C. Lorcé, H. Moutarde, P. Sznajder, A. Trawiński, and J. Wagner, Eur. Phys. J. C 81, 300 (2021).

[20] V. D. Burkert, L. Elouadrhiri, and F.X. Girod, arXiv:2104.02031.

[21] P. E. Shanahan and W. Detmold, Phys. Rev. Lett. 122, 072003 (2019).

[22] S. Meissner, A. Metz, and M. Schlegel, J. High Energy Phys. 08 (2009) 056.

[23] M. Burkardt, Int. J. Mod. Phys. A 18, 173 (2003).

[24] G. A. Miller, Phys. Rev. Lett. 99, 112001 (2007).

[25] G. A. Miller, Phys. Rev. C 80, 045210 (2009).

[26] G. A. Miller, Phys. Rev. C 99, 035202 (2019).

[27] R. L. Jaffe, Phys. Rev. D 103, 016017 (2021).

[28] C. Lorcé, Phys. Rev. Lett. 125, 232002 (2020).

[29] M. Hillery, R. F. O'Connell, M. O. Scully, and E. P. Wigner, Phys. Rep. 106, 121 (1984).

[30] J. Y. Panteleeva and M. V. Polyakov, Phys. Rev. D 104, 014008 (2021).

[31] J.-Y. Kim and H.-C. Kim, Phys. Rev. D 104, 074019 (2021).
[32] S. J. Brodsky, H.-C. Pauli, and S. S. Pinsky, Phys. Rep. 301, 299 (1998).

[33] S. Weinberg, Phys. Rev. 150, 1313 (1966).

[34] J. F. Gunion, S. J. Brodsky, and R. Blankenbecler, Phys. Rev. D 8, 287 (1973).

[35] R. N. Bracewell, The Fourier Transform and Its Applications (McGraw-Hill, New York, 2000).

[36] A. Rajan, T. Gorda, S. Liuti, and K. Yagi, arXiv:1812 .01479 .

[37] G. A. Miller and S. J. Brodsky, Phys. Rev. C 102, 022201 (2020).

[38] M. Burkardt, Phys. Rev. D 62, 071503 (2000); 66, 119903 (E) (2002).

[39] Z. Abidin and C.E. Carlson, Phys. Rev. D 78, 071502 (2008).

[40] R. Dashen and M. Gell-Mann, Phys. Rev. Lett. 17, 340 (1966).

[41] M. V. Polyakov and C. Weiss, Phys. Rev. D 60, 114017 (1999).

[42] M. Diehl, T. Feldmann, R. Jakob, and P. Kroll, Nucl. Phys. B596, 33 (2001); B605, 647(E) (2001).

[43] A. Freese and G. A. Miller, Phys. Rev. D 104, 014024 (2021).

[44] C. E. Carlson and M. Vanderhaeghen, Eur. Phys. J. A 41, 1 (2009).

[45] P. A. M. Dirac, Rev. Mod. Phys. 21, 392 (1949).

[46] T. D. Newton and E. P. Wigner, Rev. Mod. Phys. 21, 400 (1949).

[47] A. J. Kalnay and B. P. Toledo, Nuovo Cimento 48, 997 (1967).

[48] M. Pavšič, Mod. Phys. Lett. A 33, 1850114 (2018).

[49] R. Sachs, Phys. Rev. 126, 2256 (1962).

[50] J. Hudson and P. Schweitzer, Phys. Rev. D 96, 114013 (2017).

[51] S. J. Brodsky and G. P. Lepage, Adv. Ser. Dir. High Energy Phys. 5, 93 (1989).

[52] L. L. Frankfurt and M. I. Strikman, Phys. Rep. 76, 215 (1981).

[53] H. A. Bethe and C. Longmire, Phys. Rev. 77, 647 (1950).

[54] D. B. Kaplan, M. J. Savage, and M. B. Wise, Phys. Rev. C 59, 617 (1999).

[55] P. L. Chung, F. Coester, and W. N. Polyzou, Phys. Lett. B 205, 545 (1988).

[56] J. Hudson and P. Schweitzer, Phys. Rev. D 97, 056003 (2018).

[57] A. Freese and I. C. Cloët, Phys. Rev. C 100, 015201 (2019). 